\title{
Macroscopic and Microscopic Activation of $\alpha 7$ Nicotinic Acetylcholine Receptors by the Structurally Unrelated Allosteric Agonist-Positive Allosteric Modulators (ago-PAMs) B-973B and GAT107
}

\author{
Marta Quadri, Sumanta Garai, Ganesh A. Thakur, Clare Stokes, Alican Gulsevin, \\ Nicole A. Horenstein, and Roger L. Papke
}

Departments of Pharmacology and Therapeutics (M.Q., C.S., R.L.P.) and Chemistry (M.Q., A.G., N.A.H.), University of Florida, Gainesville, Florida; and Department of Pharmaceutical Sciences, Northeastern University, Boston, Massachusetts (S.G., G.A.T.)

Received June 11, 2018; accepted October 18, 2018

\section{ABSTRACT}

B-973 is an efficacious type II positive allosteric modulator (PAM) of $\alpha 7$ nicotinic acetylcholine receptors that, like 4BP-TQS and its active isomer GAT107, can produce direct allosteric activation in addition to potentiation of orthosteric agonist activity, which identifies it as an allosteric activating (ago)-PAM. We compared the properties of B-973B, the active enantiomer of B-973, with those of GAT107 regarding the separation of allosteric potentiation and activation. Both ago-PAMs can strongly activate mutants of $\alpha 7$ that are insensitive to standard orthosteric agonists like acetylcholine. Likewise, the activity of both ago-PAMs is largely eliminated by the M254L mutation in the putative transmembrane
PAM-binding site. Allosteric activation by B-973B appeared more protracted than that produced by GAT107, and B-973B responses were relatively insensitive to the noncompetitive antagonist mecamylamine compared with GAT107 responses. Similar differences are also seen in the single-channel currents. The two agents generate unique profiles of full-conductance and subconductance states, with B-973B producing protracted bursts, even in the presence of mecamylamine. Modeling and docking studies suggest that the molecular basis for these effects depends on specific interactions in both the extracellular and transmembrane domains of the receptor.

\section{Introduction}

Nicotine and $\alpha$-bungarotoxin ( $\alpha$-BTX) bind with high affinity to two different populations of receptors in the brain (Clarke et al., 1985; Jones et al., 1999). The molecular cloning of nicotinic acetylcholine receptor (nAChR) subunits led to identification of the brain receptors with high affinity for nicotine as protein complexes composed of $\alpha$ and $\beta$ subunits (Wada et al., 1988) containing primarily $\alpha 4$ and $\beta 2$ subunits (Wada et al., 1989). The $\alpha$-BTX binding sites in the brain were later shown to be associated with $\alpha 7$ subunits, which can form functional homomeric receptors (Couturier et al., 1990).

This research was supported by the National Institutes of Health [Grants R01-GM57481 and RO1-EY024717].

https://doi.org/10.1124/mol.118.113340.

S This article has supplemental material available at molpharm. aspetjournals.org.
Initial attempts to record $\alpha$-BTX-sensitive nicotinic currents from peripheral neurons were largely unsuccessful until efforts were made to achieve especially fast agonist applications (Zhang et al., 1994), something that was later understood to be due to the particularly rapid desensitization of $\alpha 7$ receptors exposed to high concentrations of agonist (Papke and Thinschmidt, 1998; Uteshev et al., 2002). Unlike other nAChRs, which produce their maximal peak current responses upon rapid saturation of all their agonist binding sites, $\alpha 7$ receptors progressively enter nonconducting states at high levels of agonist occupancy so that peak currents occur in a narrow range of agonist concentration and often occur before fast drug application is complete (Papke and Porter Papke, 2002; Williams et al., 2012). The unique desensitization properties of $\alpha 7$ receptors fundamentally limit the ionchannel currents that can be produced by typical agonists; however, the existence of the receptor's unusual desensitized conformations has also made $\alpha 7$ receptors uniquely sensitive

ABBREVIATIONS: ABNR, Adopted Basis Newton-Raphson; ACh, acetylcholine; ago-PAM, allosteric activating positive allosteric modulator; 4BPTQS, 4-(4-bromophenyl)-3a,4,5,9b-tetrahydro-3H-cyclopenta[c]quinoline-8-sulfonamide; B-973B, (3-(3,4-difluorophenyl)-N-(1-(6-(4-(pyridin-2-yl) piperazin-1-yl)pyrazin-2-yl)ethyl)propenamide); $\alpha$-BTX, $\alpha$-bungarotoxin; CAP, cholinergic anti-inflammatory pathway; CTPE, constant pressure constant temperature ensemble; DAA, direct allosteric activation; $D_{i}, P A M$-insensitive desensitization; $D_{s}$, PAM-sensitive desensitization; DMEM, Dulbecco's modified Eagle's medium; ECD, extracellular domain; GAT107, ((3aR,4S,9bS)-4-(4-bromophenyl)-3a,4,5,9b-tetrahydro-3H-cyclopenta[c]quinoline-8-sulfonamide); IFD, induced-fit docking; M2, second transmembrane domain; M3, third transmembrane domain; M4, fourth transmembrane domain; MD, molecular dynamics; nAChR, nicotinic acetylcholine receptor; PAMs, positive allosteric modulators; PNU-120596 (1(5-chloro-2, 4-dimethoxyphenyl)-3-(5-methylisoxazol-3-yl)-urea); QX-222, 2-[(2,6-dimethylphenyl)amino]-N,N,N-trimethyl-2-oxoethaniminium chloride; TMD, transmembrane domain; TQS, 3a,4,5,9b-tetrahydro-4-(1-naphthalenyl)-3H-cyclopentan[c]quinoline-8-sulfonamide. 
to selective positive allosteric modulators (PAMs) (Williams et al., 2011c), some of which eliminate the limitations imposed by desensitization by converting desensitized receptors into receptors that can show particularly long bursts of openings (Williams et al., 2011b, 2012). Efficacious type II PAMs (Grønlien et al., 2007), like PNU-120596, perturb the stability of agonistinduced desensitization by binding to a site within the receptor's transmembrane domain (TMD) (Young et al., 2008). In the absence of agonist, application of a PAM to wild-type $\alpha 7$ receptors will not produce activation but can prime receptors to generate a large potentiated response to a subsequent agonist application. The duration of such priming effects depends on the specific PAM and the concentration applied (Williams et al., 2011b; Gill et al., 2012; Papke et al., 2014b).

Whereas typical PAMs, like PNU-120596 and TQS (Grønlien et al., 2007), produce enhanced activation through coupling the transmembrane PAM site with binding of orthosteric agonists, other agents structurally related to TQS produce activation without the requirement of an orthosteric agonist (Gill et al., 2011, 2012; Gill-Thind et al., 2015), identifying them as ago-PAMs. One of the most studied ago-PAMs is GAT107, the active isomer of 4BP-TQS (Papke et al., 2014b, 2018; Bagdas et al., 2016; Horenstein et al., 2016). Several lines of evidence indicate that the direct allosteric activation (DAA) produced by GAT107, when applied without agonist, relies on binding to both the transmembrane PAM site and a novel DAA binding site in the extracellular domain (ECD) (Horenstein et al., 2016). Whereas dissociation of GAT107 from the DAA site is relatively rapid, receptors remained primed for potentiated activation by orthosteric agonists for a prolonged period (Papke et al., 2018).

Numerous ago-PAMs and allosteric antagonists have been reported that are structurally related to TQS (Gill-Thind et al., 2015; Horenstein et al., 2016). The recent report on B-973 (Post-Munson et al., 2017), an ago-PAM structurally unrelated to TQS, offers the opportunity to explore both the common features and the differences in the allosteric activation of $\alpha 7$ produced by these agents. In this study, we expanded on the macroscopic characterization of B-973 using the active isomer B-973B (Garai et al., in press) for comparison with published studies of GAT107. We also studied the activity of these two agents on the level of single-channel currents. Efficacious PAMs, like PNU-120596, in combination with agonists, induced protracted bursts of channel activation (Williams et al., 2011b, 2012; Andersen et al., 2016) with both full-conductance and subconductance states, and initial characterizations of 4BP-TQS and GAT107 single-channel activity showed similar results (Pałczyńska et al., 2012; Horenstein et al., 2016). We additionally investigated the effects of noncompetitive $\mathrm{nAChR}$ antagonists on the macroscopic and microscopic currents stimulated by the ago-PAMS.

By evaluating our results in the context of high-resolution molecular models, the present studies provided the opportunity for new insights into the molecular mechanisms of receptor function and new avenues for future drug development.

\section{Materials and Methods}

Chemicals. Solvents and reagents were purchased from Sigma-Aldrich (St. Louis, MO). Cell- culture supplies were from Life Technologies (Carlsbad, CA). Dulbecco's modified Eagle's medium (DMEM) was from Corning (Tewksbury, MA). Fetal bovine serum was purchased from Omega Scientific (Tarzana, CA). Antibiotics O-2-amino2,7-dideoxy- $D$-glycero- $\alpha$ - $D$-gluco-heptopyranosyl-( $1 \rightarrow 4)$-O-[3-deoxy4-C-methyl-3-(methylamino)- $\beta$ - $L$-arabinopyranosyl-(1 $\rightarrow 6$ )]-2-deoxy- $D$ streptamine disulfate (G418) and hygromycin B were from GIBCO and Invitrogen (Thermo Fisher, Waltham, MA), respectively. The Hank's balanced salt solution (HBSS) (Life Technologies) contained the following components expressed in millimolars: $1.26 \mathrm{CaCl}_{2}, 0.493$ $\mathrm{MgCl}_{2}, 0.407 \mathrm{MgSO}_{4}, 5.33 \mathrm{KCl}, 0.441 \mathrm{KH}_{2} \mathrm{PO}_{4}, 4.17 \mathrm{NaHCO}_{3}, 137.93$ $\mathrm{NaCl}, 0.338 \mathrm{Na}_{2} \mathrm{HPO}_{4}$, and $5.56 \mathrm{D}$-glucose. EDTA was purchased from Sigma-Aldrich. GAT107 ((3aR,4S,9bS)-4-(4-bromophenyl)-3a,4,5,9btetrahydro-3H-cyclopenta[c]quinoline-8-sulfonamide) was synthesized as described previously (Kulkarni and Thakur, 2013; Thakur et al., 2013). B-973B (3-(3,4-difluorophenyl)-N-(1-(6-(4-(pyridin-2-yl) piperazin-1-yl)pyrazin-2-yl)ethyl)propenamide) was synthesized by Dr. Ganesh Thakur as previously described (Garai et al., 2018). Mecamylamine ((1S,2R,4R)-N,2,3,3-tetramethylbicyclo[2.2.1]heptan2 -amine) and tetracaine were purchased from Sigma. PNU-120596 (1-(5-chloro-2,4-dimethoxyphenyl)-3-(5-methylisoxazol-3-yl)-urea) was synthesized by Dr. Jingyi Wang and Dr. Kinga Chojnacka as described previously (Williams et al., 2011b). Fresh ACh stock solutions were made each day of experimentation. PNU-120596, GAT107, and B-973B stock solutions were prepared in dimethylsulfoxide (DMSO), stored at $-20^{\circ} \mathrm{C}$, and used for up to a year. PNU-120596, GAT107, $\mathrm{B}-973 \mathrm{~B}$, and mecamylamine solutions were prepared freshly at the desired concentration from the stored stock solutions and dissolved in the internal solution containing (in millimolars) $165 \mathrm{NaCl}, 5 \mathrm{KCl}$, $2 \mathrm{CaCl}_{2}, 5$ HEPES (4-(2-hydroxyethyl)-1-piperazineethanesulfonic acid; Sigma Aldrich), 0.001 atropine and $10 \mathrm{D}$-glucose, $\mathrm{pH}$ 7.3. Tested solutions contained either $10 \mu \mathrm{M}$ of GAT107 or $10 \mu \mathrm{M}$ B-973B in internal solution, alone or with $30 \mu \mathrm{M}$ mecamylamine.

Heterologous Expression of $\alpha 7$ nAChRs in Xenopus Oocytes. The cDNA clones of human $\alpha 7 \mathrm{nAChR}$ and human resistance-to-cholinesterase 3 (RIC-3) were provided by Dr. Jon Lindstrom (University of Pennsylvania, Philadelphia, PA) and Dr. Millet Treinin (Hebrew University, Jerusalem, Israel), respectively. After linearization and purification of the plasmid cDNAs, RNAs were prepared using the mMessage mMachine in vitro RNA transfection kit (Ambion, Austin, TX). Alpha7 mutants were made using the QuikChange SiteDirected Mutagenesis Kit (Agilent Technologies, Santa Clara, CA) as described (Papke et al., 2011). The Y93C and C190S mutants were made in $\alpha 7 \mathrm{C} 116 \mathrm{~S}$ to ensure no spurious disulfide bonding, and the C116S mutation had no effect on $\alpha 7$ expression or responses compared with wild-type $\alpha 7$ (Papke et al., 2011).

Oocytes were surgically removed from mature female Xenopus laevis frogs (Nasco, Ft. Atkinson, WI) and injected with RNAs of $\alpha 7$ nAChR and RIC-3 as described previously (Papke and Stokes, 2010). The RIC-3 chaperone protein can improve and accelerate $\alpha 7$ expression with no effects on the pharmacologic properties of the receptors (Halevi et al., 2003). Frogs were maintained in the animal care service facility of the University of Florida, and all procedures were approved by the University of Florida Institutional Animal Care and Use Committee. All studies were carried out in accordance with the Guide for the Care and Use of Laboratory Animals as adopted and promulgated by the U.S. National Institutes of Health. In brief, the frog was first anesthetized for 15-20 minutes in 1.5-liter frog tank water containing $1 \mathrm{~g}$ of 3-aminobenzoate methanesulfonate (MS-222) buffered with sodium bicarbonate. The harvested oocytes were treated with $1.4 \mathrm{mg} / \mathrm{ml}$ collagenase (Worthington Biochemicals, Freehold, NJ) for 3 hours at room temperature in a calcium-free Barth's solution $\left(88 \mathrm{mM} \mathrm{NaCl}, 1 \mathrm{mM} \mathrm{KCl}, 2.38 \mathrm{mM} \mathrm{NaHCO}_{3}, 0.82 \mathrm{mM} \mathrm{MgSO}_{4}, 15 \mathrm{mM}\right.$ HEPES, and $12 \mathrm{mg} / \mathrm{l}$ tetracycline, $\mathrm{pH}$ 7.6) to remove the follicular layer. Stage 5 oocytes were subsequently isolated and injected with $50 \mathrm{nl}$ of 6-ng $\alpha 7 \mathrm{nAChR}$ subunit cRNA and $3 \mathrm{ng}$ RIC-3 cRNA. Recordings were carried out 1-7 days after injection.

Two-Electrode Voltage-Clamp Electrophysiology of Oocytes. Experiments were conducted using OpusXpress 6000A (Molecular Devices, Union City, CA) (Papke and Stokes, 2010). Both the voltage and current electrodes were filled with $3 \mathrm{M} \mathrm{KCl}$. Oocytes were voltage-clamped 
at $-60 \mathrm{mV}$. The oocytes were bath-perfused with Ringer's solution $(115 \mathrm{mM}$ $\mathrm{NaCl}, 2.5 \mathrm{mM} \mathrm{KCl}, 1.8 \mathrm{mM} \mathrm{CaCl}_{2}, 10 \mathrm{mM}$ HEPES, and $1 \mu \mathrm{M}$ atropine, $\mathrm{pH}$ 7.2 ) with a flow rate of $2 \mathrm{ml} / \mathrm{min}$. To evaluate the effects of experimental compounds on ACh-evoked responses of $\alpha 7 \mathrm{nAChRs}$ expressed in oocytes, two initial control responses to applications of ACh were recorded before test applications of experimental drugs alone or coapplied with the ACh. The agonist solutions were applied from a 96-well plate via disposable tips, and the drugs were either coapplied with ACh by the OpusXpress pipette delivery system or bath-applied using the OpusXpress system to switch the running buffer. Drug applications were 12 seconds long, followed by a 181-second washout period, and usually alternated between controls and test solutions. The control concentration of ACh was $60 \mu \mathrm{M}$ for wild-type $\alpha 7$. After experimental drug applications, follow-up control applications of $\mathrm{ACh}$ were made to determine primed potentiation, desensitization, or rundown of the receptors.

Data were collected at $50 \mathrm{~Hz}$, filtered at $20 \mathrm{~Hz}$, and analyzed by Clampfit 9.2 or 10.0 (Molecular Devices) and Excel (Microsoft, Redmond, WA). Data were expressed as means \pm S.E.M. from at least five oocytes for each experiment, and plotted with Kaleidagraph 4.5.2 (Abelbeck Software, Reading, PA). Concentration response data were fit to the Hill equation using the Levenberg-Marquardt algorithm. Multicell averages were calculated for comparisons of complex responses. Averages of the normalized data were calculated for each of the 10,322 points in each of the 206.44-second traces (acquired at $50 \mathrm{~Hz}$ ), as well as the S.E. for those averages.

Single-Channel Patch-Clamp Electrophysiology of Cultured Cells. The A7R3HC10 cells stably expressing human $\alpha 7$ and human RIC-3 were generated from low-passage-number human embryonic kidney (HEK) 293 cells obtained from American Type Culture Collection (Manassas, VA) as previously described (Williams et al., 2012). The A7R3HC10 cells were routinely cultured in DMEM supplemented with $10 \%$ fetal bovine serum in the presence of $0.45 \mathrm{mg} / \mathrm{ml} \mathrm{O}$-2-amino-2,7-dideoxy$D$-glycero- $\alpha-D$-gluco-heptopyranosyl-(1 $\rightarrow 4$ )-O-[3-deoxy-4-C-methyl3 -(methylamino)- $\beta$ - $L$-arabinopyranosyl-( $1 \rightarrow 6)]-2$-deoxy- $D$-streptamine disulfate (G418) and $0.015 \mathrm{mg} / \mathrm{ml}$ hygromycin $\mathrm{B}$ at $37^{\circ} \mathrm{C}$ with $5 \% \mathrm{CO}_{2}$. For normal passaging, cells were dissociated with $1 \mathrm{mM}$ EDTA in calcium-free and magnesium-free HBSS to avoid nonselective damage to the $\alpha 7 \mathrm{nAChRs}$ expressed on the cell surface. Cells with fewer than 50 passages after stable transfection were used for patch-clamp recordings. For cell-attached patch-clamp experiments, cells were plated on poly-D-lysine-coated coverslips, cultured in DMEM supplemented with $5 \%$ or $10 \%$ fetal bovine serum in the presence of $0.45 \mathrm{mg} / \mathrm{ml} \mathrm{G} 418$ and $0.015 \mathrm{mg} / \mathrm{ml}$ hygromycin B at $37^{\circ} \mathrm{C}$ with $5 \%$ $\mathrm{CO}_{2}$, and used 1-10 days after plating.

Cell-Attached Patch-Clamp Electrophysiology. Single-channel currents were recorded in the cell-attached configuration at room temperature using an MultiClamp 700B amplifier (Molecular Devices) as described previously (Horenstein et al., 2016). A solution containing (millimolars) $165 \mathrm{NaCl}, 5 \mathrm{KCl}, 2 \mathrm{CaCl}_{2}, 10$ D-glucose, and 5 HEPES, and $1 \mu \mathrm{M}$ atropine, with $\mathrm{pH}$ adjusted to 7.3 , with $\mathrm{NaOH}$ was used for the bath (external solution) and pipette-filling (internal solution). Borosilicate glass-patch pipettes (Sutter Instrument, Novato, CA) were freshly pulled using a P-97 micropipette puller to a tip diameter of 1 to $2 \mu \mathrm{m}$, firepolished, and coated with SigmaCote (Sigma-Aldrich). Patch pipettes were then back-filled with the appropriate drug dissolved in the abovementioned internal solution and had a resistance of 1.5-10 $\mathrm{M} \Omega$. Recordings were low-pass-filtered to $6 \mathrm{kHz}$ with the built-in amplifier filter (four-pole Bessel) and digitized at $50 \mathrm{kHz}$ with a DigiData 1440A (Molecular Devices) with Clampfit 10.3.0.2 (Molecular Devices). Additional analysis was conducted with QuB 1.5.0.39 (University at Buffalo, Buffalo, NY). Multiple recordings for each experimental condition were obtained from recording dates. For single-channel experiments, cellattached recordings were analyzed if on-cell seal access resistances before recording were greater than $1 \mathrm{G} \Omega$. In cell-attached patches, single-channel events were recorded at 0,10 , and $20 \mathrm{mV}$ pipette potentials from a pipette holding potential of $-60 \mathrm{mV}$.

Data from at least four individual patches for each condition were pooled to obtain enough number of events for analysis. Portions of data traces containing only single-channel activity were selected for analysis, and any section of recording with two or more channels opened simultaneously was excluded from the analysis. To determine intraburst closure, subconductance, and open durations, 1631 protracted bursts (584 in GAT107 and 1047 in B-973B) were selected at random for careful idealization. Burst analysis was conducted to define groups of one or more apparent channel openings and closures that arose from an individual channel. Single bursts were defined by closing time greater than $t_{\text {crit }}$, a critical parameter that defines the interval that separates closures within a burst (intraburst closures) from closures between different bursts (interburst closures). In our analysis, we set the $t_{\text {crit }}$ to 50 milliseconds, 5 -fold longer than the longest time constant in the intraburst closed time distribution so that less than $1 \%$ of these interburst closures would have been misidentified. All traces were filtered at $6 \mathrm{kHz}$, corrected for baseline drift, and analyzed with Clampfit 10.3.0.2 (Molecular Devices). Data traces were idealized within $\mathrm{QuB}$ using the segmental $k$-means method (Qin, 2004) and were idealized at a bandwidth of $6 \mathrm{kHz}$. After the automated idealization, the fit was manually inspected event by event, and corrections were made as necessary to the idealization. A temporal resolution limit of $1.3 \times$ filter rise time was set at 40 microseconds and imposed in all analyses (sampling rate, $50 \mathrm{kHz}$; interval, 20 microseconds). The probability of channel opening was determined according to the equation reported by Williams et al. (2011b). The mean current amplitudes measured at potentials of 0 , 10 , and $20 \mathrm{mV}$ relative to the cells' intrinsic resting potentials were used to generate I-V plots and estimate single-channel conductances. I-V curves were plotted by Kalidagraph 4.1.1, and data were represented as the mean \pm S.E.M. of five cells.

Amplitude dwell-time distribution histograms were generated for each event (i.e., closings, openings, and subconductances) and fitted with an appropriate number of Gaussian components. To exclude brief events that could not be properly resolved by our analysis owing to the limit of detection of the filter applied, we discarded dwell-time values shorter than 0.3 millisecond. We calculated the burst-length distributions and the average burst length and identified three major classes of channel openings, namely, full openings and two levels of subconductances. To discriminate between different classes of subconductances, we applied the following criteria and grouped similar subconductances accordingly: for each potential $(0,10$, or $20 \mathrm{mV})$, relative full-opening average amplitude was set to $100 \%$ and named "large open." The average of the smallest subconductances detected was $25 \%$ of the full-channel-opening amplitude, and the corresponding openings were identified as small open. A second group of subconductances was determined by opening amplitudes $50 \%$ of the full conductance and named "intermediate open." Three distinct groups of openings were therefore detected and analyzed separately. Statistical analysis was done using the unpaired $t$ test with equal variances. All differences were considered significant at $P<0.05$.

Homology Modeling of the $\alpha 7$ ECD. The $\alpha 7 \mathrm{nAChR}$ homology model was generated using the Prime module of the Schrödinger 20142 Suite (Jacobson et al., 2002, 2004). The epibatidine-bound $\alpha 7$-AChBP chimera protein structure (PDB ID: 3SQ6) (Li et al., 2011) was used as the homology model template, and the ECD of the mature human $\alpha 7$ subunit sequence was used as the model template (Uniprot P36544). Nonconserved residues were optimized based on energy minimization, and loops smaller than five residues were refined by Prime. Subunit symmetry was not enforced while generating the model. The mature protein $\alpha 7$ numbering was used to number the residues, and the five subunits were named A, B, $\mathrm{C}, \mathrm{D}$, and $\mathrm{E}$ in a counterclockwise fashion when viewing the receptor from above on the extracellular side.

Generation of Ligand Parameters. All quantum mechanics calculations for epibatidine, GAT107, TQS, and B-973B were run using Gaussian 09 (Frisch et al., 2016). The ligand molecules we investigated were drawn using Molview or Molden 5.0 (Schaftenaar and Noordik, 2000). Stereochemistry was considered for GAT107, but it was omitted for TQS and B-973B. These structures were then subjected to a two-step geometry optimization procedure, an HF/6-31G* optimization, followed by a B3LYP/6-31G** optimization. Electrostatic potentials of the optimized structures were calculated 
by single-point calculations at the HF/6-31G* level. The atomic charges were generated via restricted electrostatic potential method using the antechamber module of AmberTools16 (Jakalian et al., 2002). Ligand parameters were generated using the tleap module with GAFF (Wang et al., 2004) as the force field. Charges and atom types assigned by tleap were manually inspected for any discrepancies. Atom names in PDB files were adapted to the nomenclature used in . mol2 file of the corresponding ligand. GAT107, TQS, and B-973B molecules were neutral, and epibatidine had an overall charge of +1 .

Molecular Dynamics of the $\alpha 7$ ECD Model. The tleap routine was used for protein parameterization. Protein residues were parametrized using ff14SB (Maier et al., 2015), and epibatidine was parametrized using GAFF force field for bonded, nonbonded, and charge terms. Hydrogens were added to protein residues under neutral conditions along with missing terminal atoms. Disulfide bond-forming Cys residues were defined by changing the names of residue CYS into CYX in the PDB file. Each subunit had two disulfide bonds between residues C128-C142 and C190-C191 based on numbering of the mature $\alpha 7$ receptor. Ten $\mathrm{Na}^{+}$ions were added to neutralize the epibatidine-bound protein system. The system was solvated in an octahedral box extending $8 \AA$ from protein residues in all directions containing 12,000 TIP3P water molecules. Hydrogen mass repartitioning was applied to nonwater molecules of the solvated parameter file.

Two-step minimization was applied where a restrained minimization was followed by an unrestrained minimization. Restrained minimization (min1) put $10 \mathrm{kcal} / \mathrm{mol} \AA^{2}$ force on nonwater atom types CA, C, O, and N; 5000 steps of steepest descent minimization were followed by 45,000 steps of Adopted Basis Newton-Raphson (ABNR) minimization or until a local minimum was reached. The resulting restart file was submitted to the unrestrained minimization step (min2), where 5000 steps of steepest descent minimization was followed by 45,000 steps of ABNR minimization or until a local minimum was reached without any restraints. The restart file from the second minimization step was used for the heating step, where the system was gradually heated from 100 to $300 \mathrm{~K}$ over 500 picoseconds with a step size of 4 femtoseconds with a restraint of $5 \mathrm{kcal} / \mathrm{mol} \AA^{2}$ force on nonwater atom types CA, C, O, and N. Langevin dynamics with a collision frequency of 5 picoseconds ${ }^{-1}$ and without pressure control was used. The SHAKE algorithm was used to constrain bonds involving hydrogens. The heated structure was used for a 500 picoseconds constant pressure constant temperature ensemble (CTPE) equilibration at $1 \mathrm{~atm}$ without restraints where other parameters were same as in the heating step. A production run at constant volume and temperature was conducted after the equilibration step for 20 nanoseconds using the same Langevin parameters as the equilibration step, except there was no pressure control for this step.

Homology Modeling of the $\alpha 7$ ECD along with Transmembrane Domain. The $\alpha 7 \mathrm{nAChR}$ homology model was generated using the Prime module of the Schrödinger 2014-2 Suite (Jacobson et al., 2002, 2004). The epibatidine-bound $\alpha 7$-AChBP chimera protein structure (PDB ID: 3SQ6) (Li et al., 2011) was used as the template for the ECD, and the $\alpha 4$ subunit of the $\alpha 4 \beta 2 \mathrm{nAChR}$ crystal structure (PDB ID: 5KXI) (Morales-Perez et al., 2016) was used as the template for the TMD to maximize homology for modeling. The M3-M4 intracellular domain sequence was omitted owing to lack of a template structure for this disordered region; so the short loop that connects the M3 and M4 helices in the $\alpha 4$ subunit of the template structure was removed from P301 to S327.

To connect the M3 and M4 helices of the homology model, the aspartate residue of the M3 helix that corresponds to the position of the D299 and the glutamate residue that corresponds to the position of the E328 in the $\alpha 4$ structure were linked through a backbone amide bond. A single subunit was generated, and it was aligned five times on the $\alpha 4 \beta 2$ structure to generate a pseudosymmetrical pentamer. The dimensions of the lipid membrane were determined using the online PPM server (Lomize et al., 2011), and the receptor structure was buried in a 1-palmitoyl-2-oleoylphosphatidylcholine lipid membrane. The lipidreceptor complex was solvated with a tetrahedral water box, the protein residues were protonated at $\mathrm{pH} 7$, and the system was brought to neutrality with $\mathrm{Na}^{+}$ions using CHARMM-GUI server (Wu et al., 2014).
Molecular Dynamics of the ECD and TMD $\alpha 7$ Model. Protein residues were parametrized according to the same method used for the $\alpha 7$ ECD model. A two-step minimization was applied, where a restrained minimization was followed by an unrestrained minimization. Restrained minimization (min 1 ) put $10 \mathrm{kcal} / \mathrm{mol} \AA^{2}$ force on nonwater atom types CA, $\mathrm{C}, \mathrm{O}$, and $\mathrm{N}$. Five thousand steps of steepest descent minimization were followed by 15,000 steps of ABNR minimization or until a local minimum was reached. The resulting restart file was submitted to the unrestrained minimization step ( $\min 2$ ), where 5000 steps of steepest descent minimization were followed by 15,000 steps of ABNR minimization or until a local minimum was reached without any restraints. The restart file from the second minimization step was used for the heating step, in which the system was gradually heated from 0 to $300 \mathrm{~K}$ over 3 nanoseconds with a step size of 4 femtoseconds with a restraint of $5 \mathrm{kcal} / \mathrm{mol} \AA^{2}$ force on nonwater atom types CA, C, O, and N. Langevin dynamics with a collision frequency of 5 picosecond ${ }^{-1}$ and without pressure control was used. The SHAKE algorithm was used to constrain bonds involving hydrogens. The heated structure was equilibrated through two 20-nanosecond CTPE calculations at $1 \mathrm{~atm}$ pressure and $300 \mathrm{~K}$ temperature first with a restraint of $2 \mathrm{kcal} / \mathrm{mol} \AA^{2}$ and then $0.5 \mathrm{kcal} / \mathrm{mol} \AA^{2}$. An CTPE MD simulation at $1 \mathrm{~atm}$ pressure and $300 \mathrm{~K}$ temperature was run for 200 nanoseconds, and the final pose of this structure was minimized for 5000 steps of steepest descent minimization, followed by 15,000 steps of ABNR minimization or until a local minimum was reached without any restraints. Only the TMD portion of this structure was used for the docking calculations.

Docking. Schrödinger 2014-2 was used for all docking calculations. The final minimized structure of the ECD or the TMD model was used as the docking model for the docking calculations with GAT107 and B-973B. For the ECD docking calculations, the GlideXP module of the Schrödinger Suite (Friesner et al., 2006) was used such that the ligand conformers were allowed, but the protein structure was rigid. Docking was done for all five interfaces of the ECD model $\left(\mathrm{I}_{\mathrm{AB}}, \mathrm{I}_{\mathrm{BC}}, \mathrm{I}_{\mathrm{CD}}\right.$, $\mathrm{I}_{\mathrm{DE}}$, and $\mathrm{I}_{\mathrm{EA}}$ ). The top 10 structures were kept for each ligand at each interface based on docking scores where the top pose had the lowest Glide score. For the TMD model docking, an initial Glide docking calculation was run, but it failed to yield an overlapping binding site for both GAT107 and B-973 or produced no poses at all, depending on the interface. As a result, an induced-fit docking (IFD) approach was used to allow optimization of the binding environment within the TM region. The IFD method involved a flexible ligand approach and allowed the protein refinement of the side chain residues within $5 \AA$ of the ligand. An implicit membrane was placed manually to cover the transmembrane helix residues, based on the prediction of the membrane residues by the PPM server. Up to 20 structures were recorded for each ligand and the corresponding protein geometry. The IFD method was used for interfaces that produced some glide poses during the first trial. The poses resulting from IFD calculations were similar for the remaining interfaces, so we used the poses at $I_{\mathrm{AB}}$ for our analyses.

\section{Results}

\section{Macroscopic Responses of Human $\alpha 7 \mathrm{nAChR}$ to B-973B}

As previously reported for the racemic compound B-973, the active enantiomer B-973B (Garai et al., 2018) is a very effective modulator of $\alpha 7$ ion channel activation. DAA was observed at concentrations of $1 \mu \mathrm{M}$ and greater, with persistent primed potentiation of subsequent $\mathrm{ACh}$ responses (Fig. 1A). It is also a potent and efficacious PAM of ACh-evoked responses (Fig. 1B). Note that once coapplied at high concentrations with ACh, currents persisted beyond the washout of the drug from the bath (Fig. 1B, lower trace). Whereas DAA (Fig. 2A) and primed potentiation (PP, Fig. 2B) were observed only at concentrations greater than $1 \mu \mathrm{M}$, significant $(P<0.001)$ PAM activity was seen with coapplication of $300 \mathrm{nM}$ B-973B compared with ACh alone (Fig. 2C). 
A
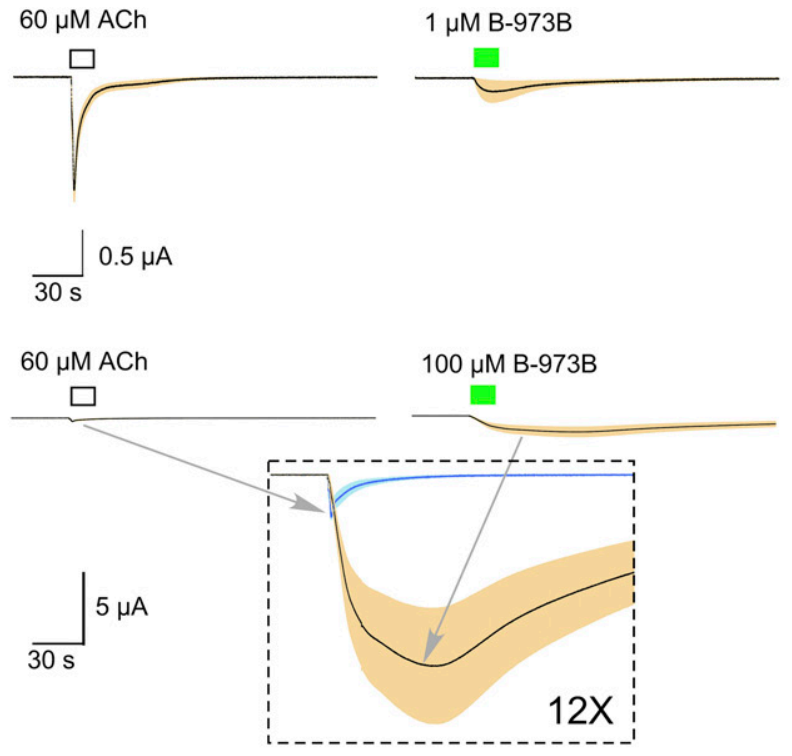

B

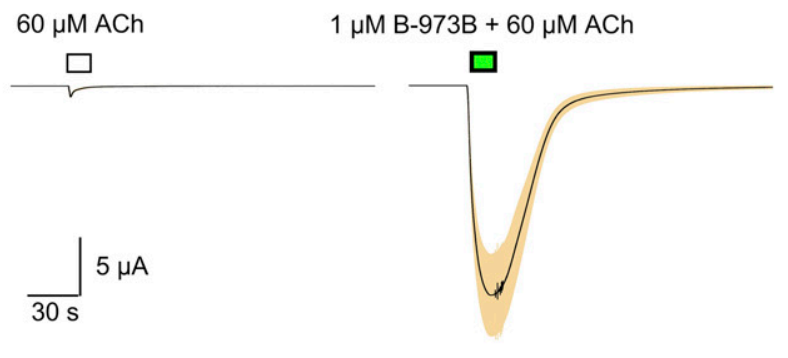

$60 \mu \mathrm{M} \mathrm{ACh}$

$100 \mu \mathrm{M} B-973 \mathrm{~B}+60 \mu \mathrm{M}$ ACh

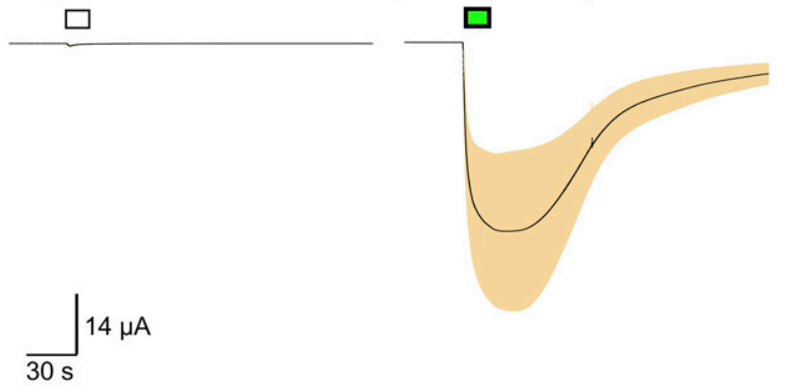

$60 \mu \mathrm{M} \mathrm{ACh}$
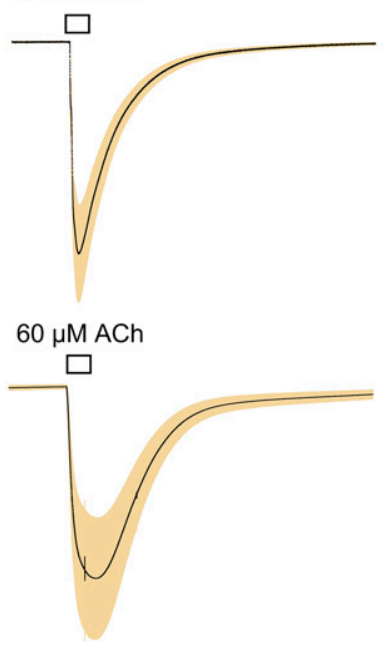

$60 \mu \mathrm{M} \mathrm{ACh}$

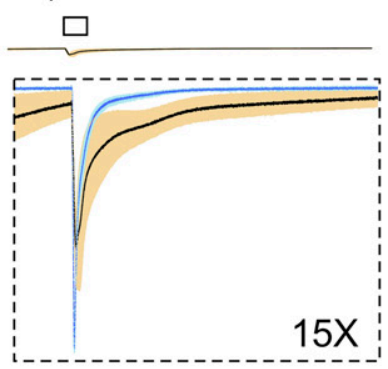

$60 \mu \mathrm{M}$ ACh

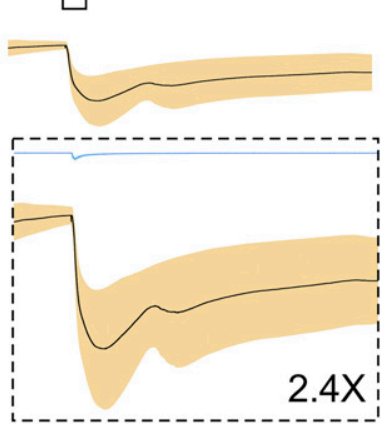

Fig. 1. Activation and potentiation of ACh-evoked responses of cells expressing human $\alpha 7$ by B-973B. (A) Averaged normalized responses (solid lines) of multiple cells $(n \geq 5) \pm$ S.E.M. (tan area) to ACh before and after application of B-973B at 1 or $100 \mu \mathrm{M}$. Each trace of 10,322 points is 206.44 seconds long. Responses of individual cells were each normalized to their responses to $60 \mu \mathrm{M} \mathrm{ACh}$ before the B-973B application. ACh responses are shown for comparison, and scale bars reflect the average $\mathrm{ACh}$ responses from each experiment. The net charge of the DAA produced by $1 \mu \mathrm{M}$ B-973B was equivalent to that of the initial $\mathrm{ACh}$ response, whereas the net charge of the primed potentiated response to $\mathrm{ACh}$ after $1 \mu \mathrm{M}$ B-973B was $7.3 \pm 17$-fold greater. The net charge of the DAA produced by $100 \mu \mathrm{M}$ B-973B was $86 \pm 25$ times that of the initial ACh response, whereas the net charge of the primed potentiated response to ACh after $1 \mu \mathrm{M}$ B-973B was $525 \pm 154$ fold larger. The data in the inset show the initial ACh controls (blue), and the $100 \mu \mathrm{M}$ DAA (black and tan) at a 12-fold increased scale to illustrate the relative size and duration of the allosterically activated responses. (B) Averaged normalized responses (solid lines) of multiple cells $(n \geq 5) \pm$ S.E.M. (tan area) to $60 \mu \mathrm{M}$ $\mathrm{ACh}$ compared with ACh responses potentiated by either 1 or $100 \mu \mathrm{M}$ B-973B and $\mathrm{ACh}$ responses after the $\mathrm{B}-973 \mathrm{~B}-\mathrm{ACh}$ coapplications. In both the upper and lower traces, the insets compare the initial ACh controls (blue) and the subsequent $\mathrm{ACh}$ responses at the increased scales indicated. Note the failure of the responses to return to baseline after coapplication of $60 \mu \mathrm{M} \mathrm{ACh}$ and $100 \mu \mathrm{M}$ B-973B.
The concentration-response data for allosteric activation and primed potentiation were not suitable to be fit to the Hill equation since the responses continued to increase over the range tested (1-100 $\mu \mathrm{M})$, and higher concentrations produced responses that were too large to be voltage-clamped. The PAM activity, however, could be fit to the Hill equation, provided the net charge was calculated with our standard protocol (Papke and Porter Papke, 2002) for just the 120-second period immediately after the start of drug delivery because, as noted already, at high concentrations, responses did not return to baseline and tended to become unstable. The $I_{\max }$ values were $51 \pm 9$ and $476 \pm 40$ (relative to $60 \mu \mathrm{M}$ ACh controls) for peak current and net charge, respectively. The $\mathrm{EC}_{50}$ values were $2.2 \pm 1.8$ and $3.6 \pm$ $1.1 \mu \mathrm{M}$ for peak current and net charge, respectively.
Shown in Fig. 3 is a comparison of the allosteric activation produced by $10 \mu \mathrm{M}$ B-973B and GAT107, along with the structures of the two active isomers. Although comparable in net charge, the GAT107 responses were more reversible than the B-973B responses, and comparison of the peak currents associated with the application of the ago-PAMs alone compared with ACh controls from the same cells indicated that peak currents during the allosteric activation phase were greater $(P<0.05)$ for GAT107 compared with B-973B. The kinetics of B-973B DAA appear slower than those of GAT107. Specifically, GAT107 activation reverses immediately upon washout, whereas B-973B activation still increases after washout begins. This may be due to slower unbinding in the ECD, different coupling to the PAM site, or both these factors. 
A

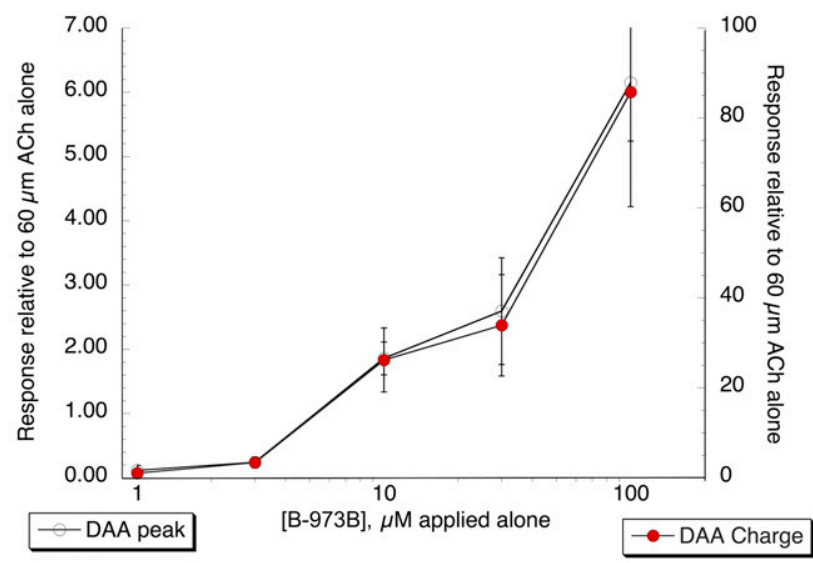

B

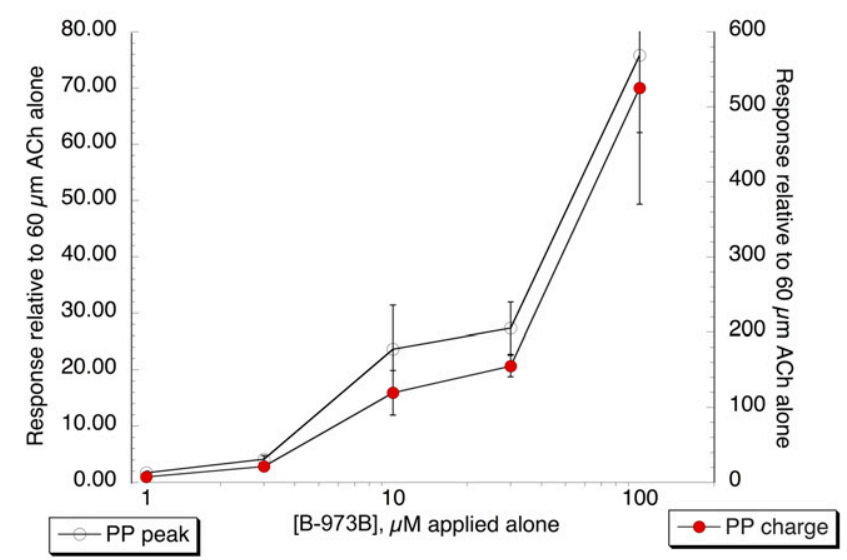

C

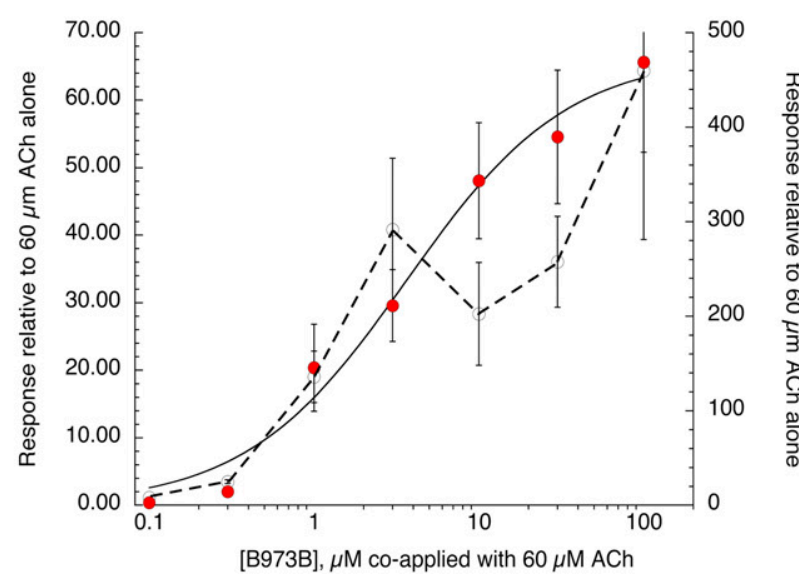

Fig. 2. Concentration-response studies of B-973B. (A) DAA of human $\alpha 7$ $\mathrm{nAChR}$ expressed in oocytes. Data represent the average ( \pm S.E.M.) responses, normalized to the average of two initial ACh controls of at least five cells at each concentration. Both the peak currents (left axis) and net charge (right axis) responses are shown. (B) Potentiated $60 \mu \mathrm{M}$ ACh responses of human $\alpha 7$ $\mathrm{nAChR}$ expressed in oocytes after application of B-973B at the concentrations indicated (primed potentiation, see Fig. 1A). Data represent the average ( \pm S.E.M.) responses, normalized to the average of two initial $\mathrm{ACh}$ controls of at least five cells at each concentration. Both the peak currents (left axis) and net charge (right axis) of the responses are shown. (C) Potentiated $60 \mu \mathrm{M}$ ACh responses of human $\alpha 7 \mathrm{nAChR}$ expressed in
Additionally, the two ago-PAMs appear to differ in the rates and manner they equilibrate activation and the PAMsensitive $\left(D_{\mathrm{s}}\right)$ and PAM-insensitive $\left(D_{\mathrm{i}}\right)$ desensitized states (Williams et al., 2011b). A prolonged application of $10 \mu \mathrm{M}$ GAT107 produced a large transient phase of activation before an equilibration toward a steady-state level of activation, as seen with ACh co-applied with PNU-120596 (Williams et al., 2011b). In contrast, activation by $10 \mu \mathrm{M}$ B-973B was more uniform over the same period (Supplemental Fig. 1).

One interesting aspect of the allosteric activation of $\alpha 7$ by GAT107 is that it can overcome the limitations to orthosteric activation imposed by various point mutations, including mutation of the key aromatic residue Y93 and elimination of the vicinal disulfide between cysteines 190 and 191 at the tip of the C-loop (Papke et al., 2014b; Horenstein et al., 2016). B-973B also effectively activated these mutants (Fig. 4A). Consistent with their allosteric activity on wild-type receptors (Fig. 3), GAT107 produced larger transient peak currents than $\mathrm{B}-973 \mathrm{~B}$, but the net-charge responses were not different.

The effects of the M254L mutation, previously shown to limit the allosteric activity of PNU-120596 (Young et al., 2008), 4BP-TQS (Gill et al., 2011), and GAT107 (Papke et al., 2014b), were evaluated with B-973B and compared with similar experiments with GAT107 (Fig. 4B). It is interesting to note that whereas this mutation was more than $90 \%$ effective at reducing the allosteric and potentiating effects of GAT107, it was essentially $100 \%$ effective on eliminating allosteric activation and potentiating effects of B-973B.

As shown in Figs. 1 and 3, cells did not recover to baseline after coapplication of $\mathrm{ACh}$ with $\mathrm{B}-973 \mathrm{~B}$ at concentrations of $\geq 10 \mu \mathrm{M}$. We have previously shown that mecamylamine is an effective inhibitor of PNU-120596- (Williams et al., 2011b) and GAT107-potentiated currents (Papke et al., 2018) with an $\mathrm{IC}_{50}$ for the inhibition of PNU-120596-potentiated currents, similarly to inhibition of responses evoked by ACh alone (Peng et al., 2013). Curiously, we observed that mecamylamine was relatively ineffective at bringing the protracted currents activated by B-973B and ACh back to baseline (Fig. 5).

The kinetics of B-973B DAA appear slower than those of GAT107. That is, GAT107 activation reversed immediately upon washout, whereas B-973B activation still increased after washout begins, possibly due to slower unbinding in the ECD, different coupling to the PAM site, or both these factors. Additionally, the two ago-PAMs appear to differ in the rates and manner that they equilibrate activation and the PAM-sensitive $\left(D_{s}\right)$ and PAM-insensitive $\left(D_{i}\right)$ desensitized states (Williams et al., 2011b). A prolonged application of $10 \mu \mathrm{M}$ GAT107 produced a large transient phase of activation

oocytes during the coapplication of $\mathrm{ACh}$ and $\mathrm{B}-973 \mathrm{~B}$ at the concentrations indicated. Data represent average ( \pm S.E.M.) responses, normalized to the average of two initial ACh controls of at least five cells under each concentration. Plotted are both the peak currents (left axis) and net charge (right axis) of the responses. The peak current data were not appropriate to fit to the Hill equation. Peak currents, in general, have limited value as measurements of $\alpha 7 \mathrm{nAChR}$ function since they are confounded by the kinetics of solution exchange and the dynamics of receptor desensitization (Papke and Porter Papke, 2002). The net-charge data were fit to the Hill equation using the Levenberg-Marquardt algorithm with a Hill coefficient of $0.9 \pm 0.2, I_{\mathrm{Max}}=476 \pm 40, n=0.89$ \pm 0.18, and $\mathrm{EC}_{50}=3.66 \pm 1.15 \mu \mathrm{M}$ 


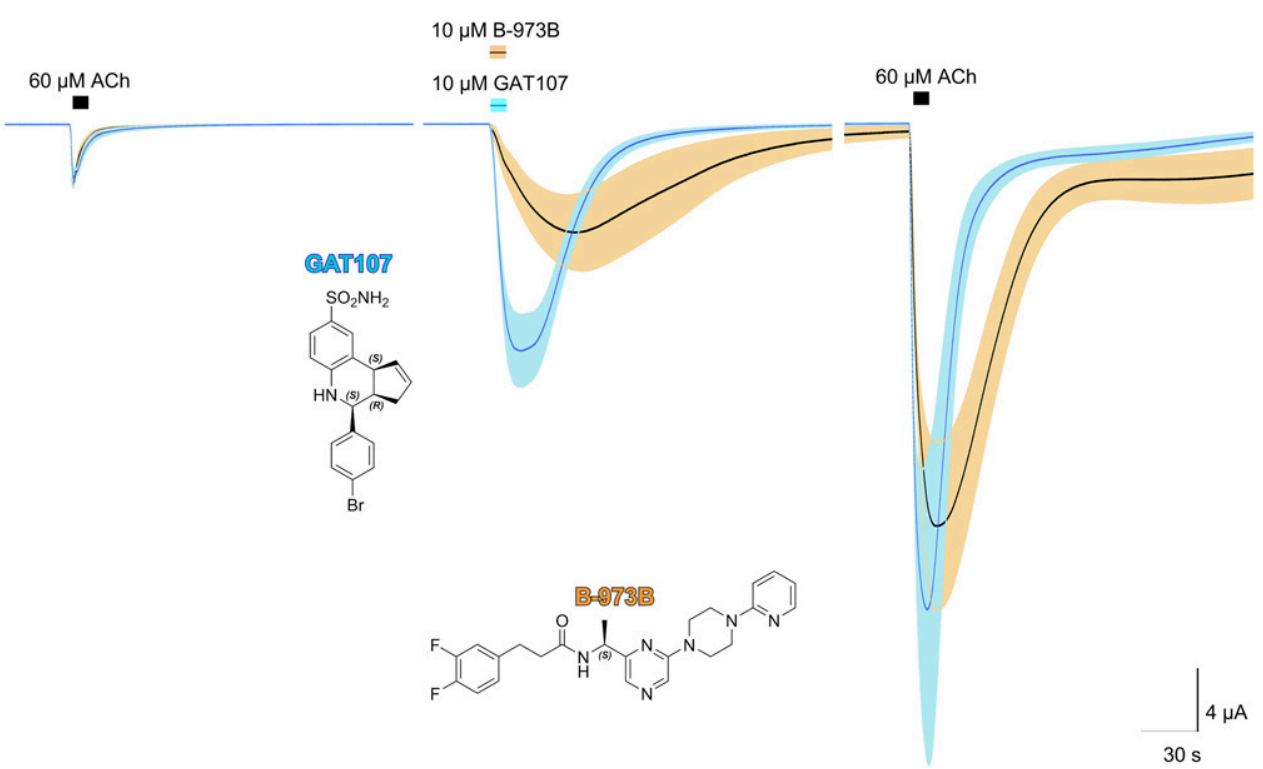

Fig. 3. Macroscopic responses of $\alpha 7$ nAChR expressed in oocytes to $10 \mu \mathrm{M}$ GAT107 (blue) or $10 \mu \mathrm{M}$ B-973B (black and $\tan$ ) compared. Shown are the averaged normalized responses (solid lines) of six cells $(n \geq 5) \pm$ S.E.M. (tan area) to the ACh prior to and following application of either $10 \mu \mathrm{M}$ GAT107 or $10 \mu \mathrm{M}$ B-973B. Each trace of 10,322 points is 206.44 seconds long. Responses of individual cells were each normalized to their responses to $60 \mu \mathrm{M}$ ACh before the agoPAM applications. The vertical scale bar is calculated based on the average of the ACh control responses. The structures of the two ago-PAMs are also shown.

before an equilibration toward a steady-state level of activation, as seen with ACh coapplied with PNU-120596 (Williams et al., 2011b). In contrast, activation by $10 \mu \mathrm{M}$ B-973B was more uniform over the same period (Supplemental Fig. 1).

\section{Microscopic (Single-Channel Currents)}

General Observations. In control experiments with untransfected HEK cells, neither GAT107 ( $n=3$, 15-minute recordings) nor $\mathrm{B}-973 \mathrm{~B}$ ( $n=3,15$-minute recordings) stimulated single-channel currents, whereas allosterically activated channel openings were observed in cell-attached patches on A7R3HC10 cells (Williams et al., 2012), and HEK cells stably transfected with $\alpha 7$ and RIC3. Openings occurred primarily in protracted bursts (Pałczyńska et al., 2012; Horenstein et al., 2016) (Fig. 6A), like those produced by PNU-120596 when coapplied with an orthosteric agonist (Williams et al., 2012; Andersen et al., 2016). The $\alpha 7$ ago-PAM activity of GAT107 and $\mathrm{B}-973 \mathrm{~B}$ resulted primarily in prolonged channel openings consisting mainly of isolated protracted bursts of several hundred milliseconds of duration, consistent with large sporadic increases in single-channel $P_{\text {open }}$, so that each burst is suitable for analysis as the behavior of one channel. We define burst as a group of openings and closures arising from an individual channel; single bursts were defined by closing time greater than the $t_{\text {crit }}$. The $t_{\text {crit }}$ is a critical parameter to define the interval separating closures within a burst (intraburst closures) from closures between different bursts (interburst closures). In our analysis, we set the $t_{\text {crit }}$ to 50 milliseconds so that every closure greater than 50 milliseconds defined a single and isolated burst in our analysis (see Materials and Methods). The $t_{\text {crit }}$ of 50 milliseconds was not applied to subconductances within big channel openings.

In general, bursts recorded in the presence of $10 \mu \mathrm{M}$ B-973B were longer than those activated by $10 \mu \mathrm{M}$ GAT107, averaging $2.14 \pm 0.08$ seconds $(n=1047)$ compared with $1.64 \pm 0.11$ seconds $(n=584)$. For both ago-PAMs, the burst-duration distributions (Fig. 6B) were best fit with three components with similar time constants (Table 1); however, the relative area of the longest component was greater for B-973B than for GAT107.
Bursts were further characterized by the intraburst components (Fig. 7; Table 2). On average, approximately 130 intraburst state conversions occurred with B-973B and 84 with GAT107. The major differences were apparent in $\alpha 7$-state stabilization and intraburst opening-time durations. For example, $80 \%$ of the large openings in B-973B were fit to a time constant of $28.2 \pm 0.08$ milliseconds and $16 \%$ to a longer component of $51.3 \pm 0.4$ milliseconds, whereas for GAT107, $23 \%$ were fit to $11.7 \pm 3.2$ milliseconds and $30 \%$ to the longest time constant of $18.9 \pm 1.7$ milliseconds. Additionally, $50 \%$ of all intraburst state conversions for B-973B were to the large open state and only $32 \%$ for GAT107 to the large open state.

B-973B also induced and stabilized prolonged subconductance $\alpha 7$ states, both within bursts of large opening and occasionally as isolated bursts without large openings. Although still able to induce subconductances, GAT107 subconductances were much less frequent and prolonged. Indeed, GAT107 subconductances appeared as brief, isolated intraburst openings and only very briefly and rarely as isolated bursts, consistent with our previous observations (Horenstein et al., 2016). Therefore, resolution and analysis of GAT107 subconductances were more challenging compared with B-973B.

Within most recordings, B-973B presented all three different openings (large open, intermediate and small subconductance openings). To discriminate between the three different openings, we subdivided them according to the amplitude (picoampere) of the currents. The intermediate and small subconductance openings were analyzed separately and as to whether they were isolated from large openings. Currentvoltage analysis of the three current levels indicated conductances of $100.9 \pm 8.0,59.5 \pm 5.8$, and $23.5 \pm 1.8 \mathrm{pS}$ for the large, intermediate, and small channel openings, respectively, with no significant differences observed between the currents stimulated by GAT107 and B-973B.

Starting from the total number of patches, for each drug, we analyzed the type of bursts present and calculated the relative percentages (Table 1). For GAT107, approximately $18 \%$ of patches analyzed contained exclusively subconductances, and $78 \%$ of the patches with large channel openings also displayed 
A Responses of non-orthosterically activatible mutants

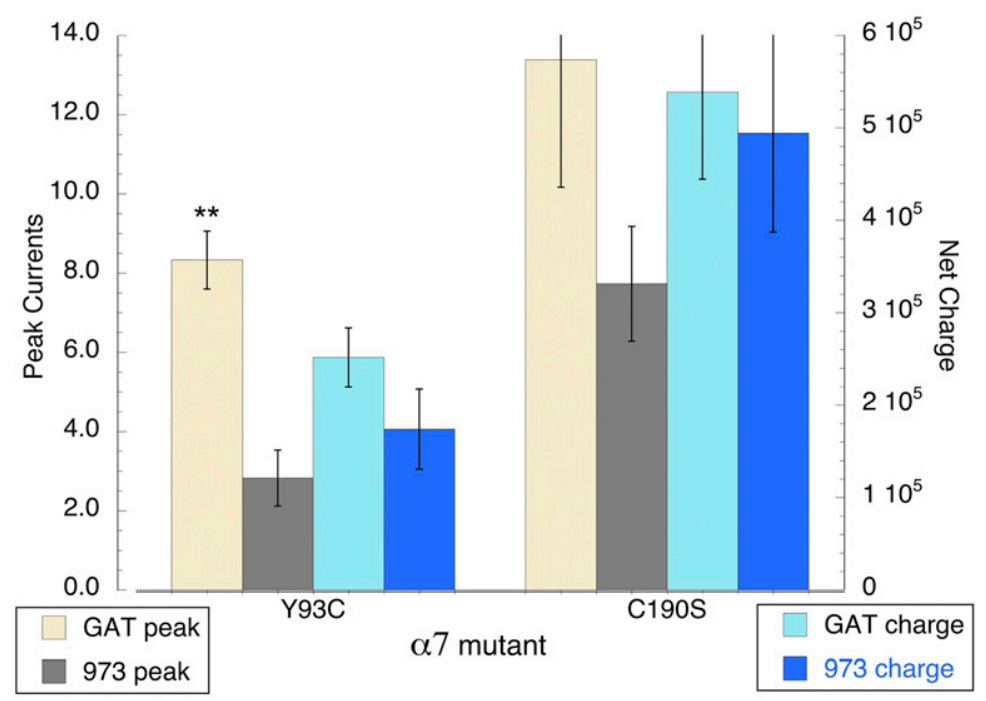

B Effects of the M254L mutation on ago-PAM activities
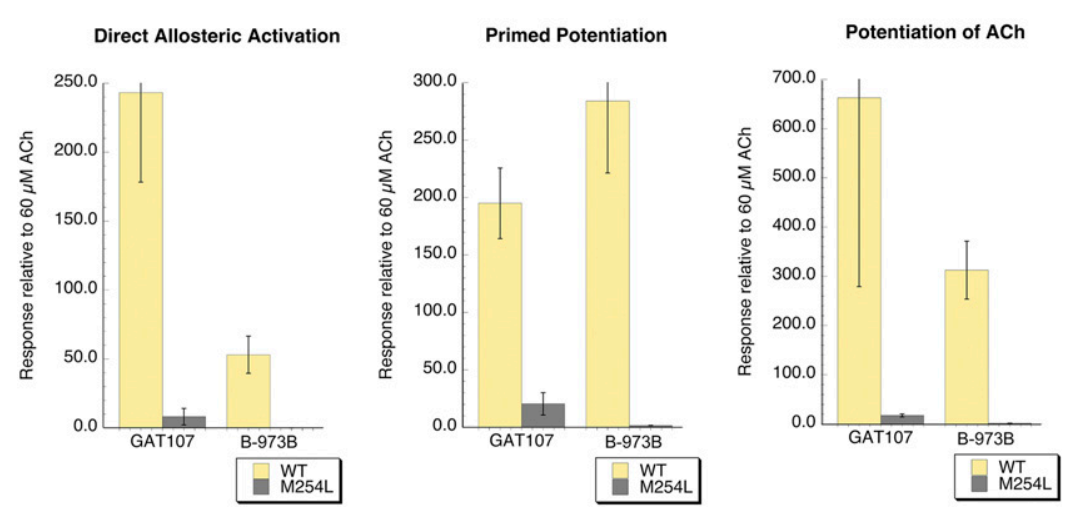

Fig. 4. Mutations in $\alpha 7$ differentially regulate the activity of orthosteric agonists and ago-PAMs. (A) The Y93C and C190S mutants of $\alpha 7$ have previously been shown to be unresponsive to ACh and other orthosteric agonists but were well activated by the ago-PAM GAT107 (Horenstein et al., 2016). Shown are the average responses of five cells $( \pm$ S.E.M.) to either $10 \mu \mathrm{M}$ GAT107 or $10 \mu \mathrm{M}$ B-973B. Although the peak current responses of the Y93C mutants to GAT107 were greaterer than the peak currents to B-973B $(* * P<0.01)$, the responses of these two mutants were otherwise comparable to both ago-PAMs. Note that the data were not normalized; the comparison was between the peak currents evoked by one ago-PAM compared with those produced by the other ago-PAM in different cells from the same injection set, recorded on the same day. (B) Regulation of allosteric activity by a mutation in the transmembrane PAM binding site. Net-charge allosteric activation and primed potentiation produced by $10 \mu \mathrm{M}$ applications are shown compared with effects in wild-type $\alpha 7$, along with responses to $60 \mu \mathrm{M}$ ACh coapplied $10 \mu \mathrm{M}$ of the ago-PAMs. Data are the averages of five or more cells ( \pm S.E.M.), normalized to the initial responses to $60 \mu \mathrm{M}$ ACh alone in the same cells. Also shown are equivalent data for $10 \mu \mathrm{M}$ GAT107 applied alone or coapplied with $60 \mu \mathrm{M}$ ACh. detectable intraburst subconductances $(0.25$ and 0.5 openings). In the case of B-973B, $30 \%$ of patches analyzed contained exclusively subconductances, and $100 \%$ of the patches with large openings also displayed intraburst subconductances ( 0.25 and 0.5 openings). Although the data suggest that for both drugs subconductances were frequent, there were notable differences. For example, only for B-973B did patches with large openings always also display subconductances.

The apparent intraburst closures were mostly comparable in duration for the two ago-PAMs ( $\sim 0.5$ millisecond) with an additional component around 2 milliseconds (Table 2), even though their frequencies were quite different. Note that for this analysis, closed-time values shorter than 0.3 millisecond were discarded to exclude brief events that could not be resolved properly. Both GAT107 and B-973B occasionally (5\%-10\% of the times) induced intraburst closures longer than 10 milliseconds, suggesting an infrequent intraburst closed state of greater stability. With our burst criteria selection of 50 milliseconds (Materials and Methods), it is likely that some of what we identified as separate bursts actually were part of the same burst; most of the interburst closed times were much longer than 50 milliseconds, but we did not investigate this in detail.
For the dwell-time analyses, we separately analyzed large openings, intermediate openings, and small openings. Additionally, we evaluated whether the intermediate openings and subconductances states occurred in isolation or within bursts containing large openings.

As noted already, on average, the long bursts of openings evoked and potentiated by $10 \mu \mathrm{M}$ GAT107 and $10 \mu \mathrm{M}$ B-973B were quite different (Fig. 7; Table 2). For GAT107, two different intraburst open times were observed that averaged $0.64 \pm 0.23$ millisecond and $18.9 \pm 1.67$ milliseconds. In contrast, the intraburst large conductance openings with B-973B were well fit by a single time constant with an average opening time duration of $28.2 \pm 0.08$ milliseconds. These data highlight significant differences in ago-PAM behavior on $\alpha 7$ nAChR, indicating that GAT107 evoked more brief and fast openings compared with B-973B, which usually induced longer large conductance openings.

We also detected significant differences in the duration and frequency of the subconductance openings evoked by the two tested compounds. Average isolated subconductance openings were fit to several time constants for GAT107 and B-973B, with larger percentages being of longer duration for B-973B (Table 2). These differences may parallel the previously 


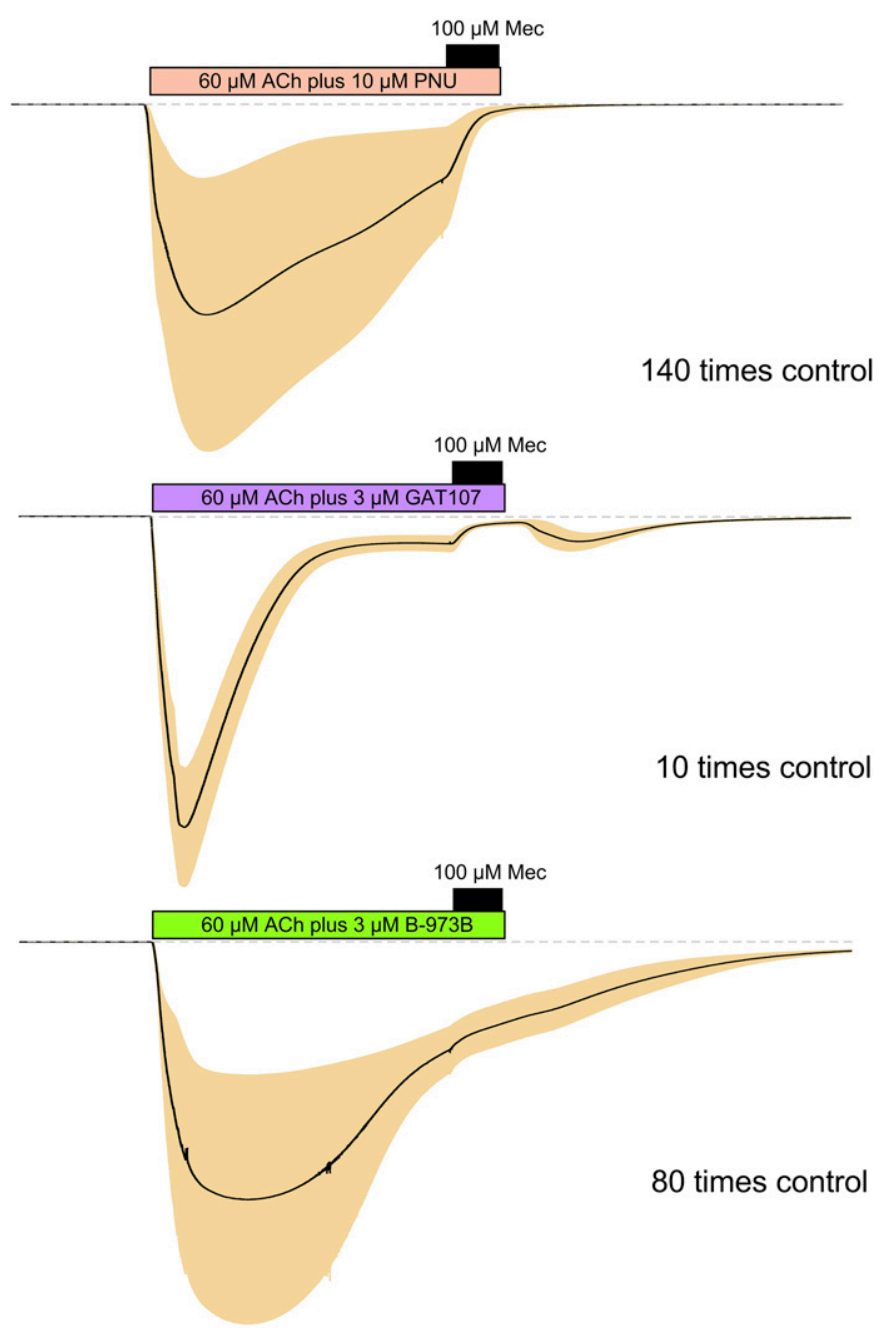

Fig. 5. Differential effects of mecamylamine on PAM-potentiated currents. Shown are the averaged macroscopic responses of cells expressing human $\alpha 7$ $\mathrm{nAChR}(n \geq 5)$ to applications of $60 \mu \mathrm{M}$ ACh plus PNU-120596, GAT107, or $\mathrm{B}-973 \mathrm{~B}$ at the indicated concentrations, normalized to the peak current amplitude of their preceding ACh controls. At the end of a 60 -second static bath incubation with $\mathrm{ACh} / \mathrm{PAM}$ combination, the drugs were coapplied with $100 \mu \mathrm{M}$ mecamylamine for 12 seconds, followed by the usual washout with Ringer's solution. The peaks and the S.E.M. envelopes are scaled as indicated relative to the preceding ACh controls (data not shown).

discussed differences in bursts of large conductance openings, suggesting that B-973B induces and stabilizes prolonged channel opening compared with GAT107.

Mecamylamine Effects on B-973B- and GAT107Evoked $\alpha \mathbf{7}$ nAChR Currents. In the presence of the neuronal nAChR antagonist mecamylamine, GAT107 and B-973B $\alpha 7$ receptor activation was affected in very different ways (Fig. 8). GAT107-stimulated currents antagonized by mecamylamine showed both large conductances and subconductances in $29 \%$ of the patches and had profoundly changed opening durations, resulting in very brief channel openings with long intraburst closing times, as evidenced by the representative traces displayed in Fig. 8A. These effects are similar to those reported by Peng et al. (2013) for mecamylamine on PNU-120596-potentiated currents, where the drug both decreased the duration of large openings and caused the appearance of a subconductance. In contrast, B-973B coapplication with mecamylamine resulted in complete abolition of large conductances of the receptor and the appearance of a novel subconductance (Fig. 8B) in bursts of increased duration (Fig. 9) compared with those recorded with B-973B alone (Fig. 6).

The short durations of the open events observed in the presence of mecamylamine made the estimation of singlechannel amplitudes difficult, especially in the case of the shortened GAT107 currents. The subconductance preserved in the presence of B-973B and mecamylamine was estimated to be $60 \pm 25 \mathrm{pS}$, consistent with the intermediate state observed in the absence of mecamylamine. The conductance levels in the presence of mecamylamine and GAT107 corresponded most closely to the large conductance and small subconductance observed in the absence of the antagonist.

The kinetics of the currents stimulated by the ago-PAMs GAT107 or B-973B in co-application with $30 \mu \mathrm{M}$ mecamylamine showed numerous differences, especially compared with currents observed when no antagonist was present. As noted, in the absence of mecamylamine, burst duration distributions were best described by three exponentials with an overall average length of 1.64 seconds for a GAT107 burst. Relatively few bursts were observed in the presence of GAT107 and mecamylamine ( $n=53$ bursts, 14 patches), and the distribution was fit with two exponentials. The overall average burst duration was reduced to 0.42 second \pm 79 milliseconds. In remarkable contrast, the B-973B bursts, which had an overall average of 2.14 seconds in the absence of mecamylamine, were increased in frequency by mecamylamine ( $n=151$ bursts, three patches) and increased in average duration to 3.08 second (Fig. $8 \mathrm{C}$ ). They were uniform in amplitude (Fig. 8A), and the distribution was fit by a single exponential ( $\tau=2.8$ second, Fig. 9). Analysis of intraburst activity also showed reduced richness of channel behavior in the presence of mecamylamine for both ago-PAMs and a special tendency to constrain the channel's behavior to long sequences of small openings in the case of B-973B-evoked activity. These dissimilarities indicate a qualitatively different mechanism for channel block, dependent not on the antagonist but rather on the specific channel activator, suggesting that the two allosteric activators provide distinctly different environments for drug binding and for the coupling of that binding to further conformational changes. The protracted bursts of small conductance openings in the presence of B-973B and mecamylamine are consistent with the sequential open channel block first described by Neher and Steinbach (1978) of 2-[(2,6-dimethylphenyl)amino]-N,N,N-trimethyl-2oxoethaniminium chloride (QX-222) and the muscle nAChR channel and are also consistent with the relative resistance of the B-973B macroscopic currents to mecamylamine (Fig. 5). The sequential open-channel block model predicts that, with shorter open times per burst and longer bursts, the total open time within a burst should be the same with and without the blocker since it represents the average time required for the blocker to dissociate. We calculated the average open and closed times across all bursts for the four conditions and calculated the $P_{\text {open }}$ within the bursts as the average open time divided by the sum of the average open and closed time (Table 3). Multiplying that value by the average burst duration gives the average open time within a burst. Averaging across all bursts, in the absence of mecamylamine, open time in B-973B bursts was 1854.3 milliseconds; in the presence of mecamylamine, the average open time per burst was 
A
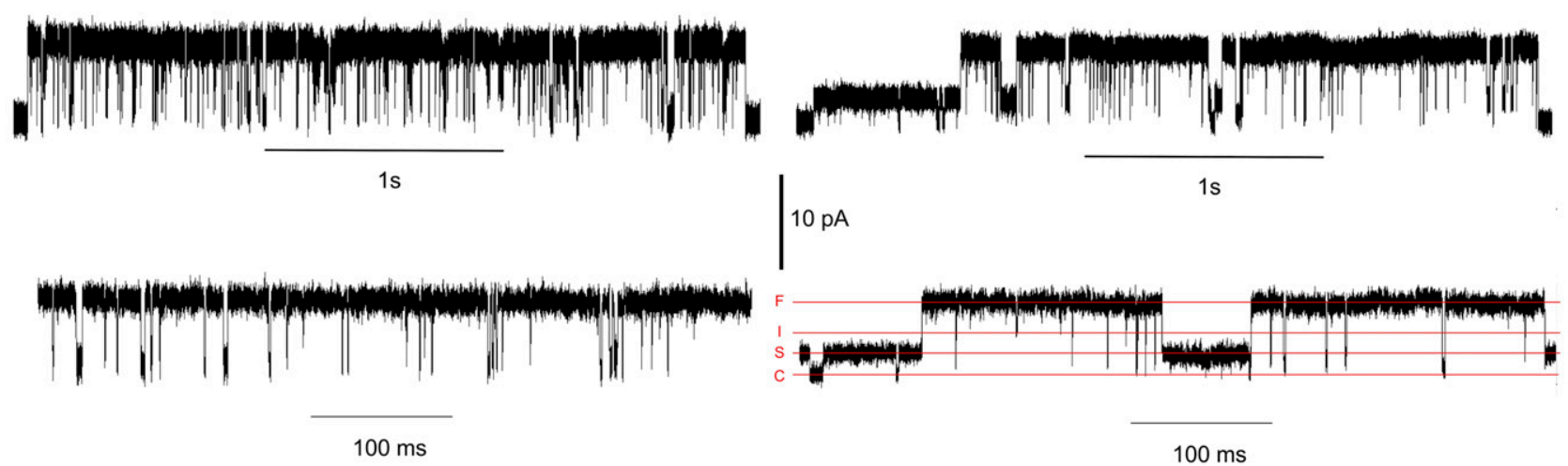

B
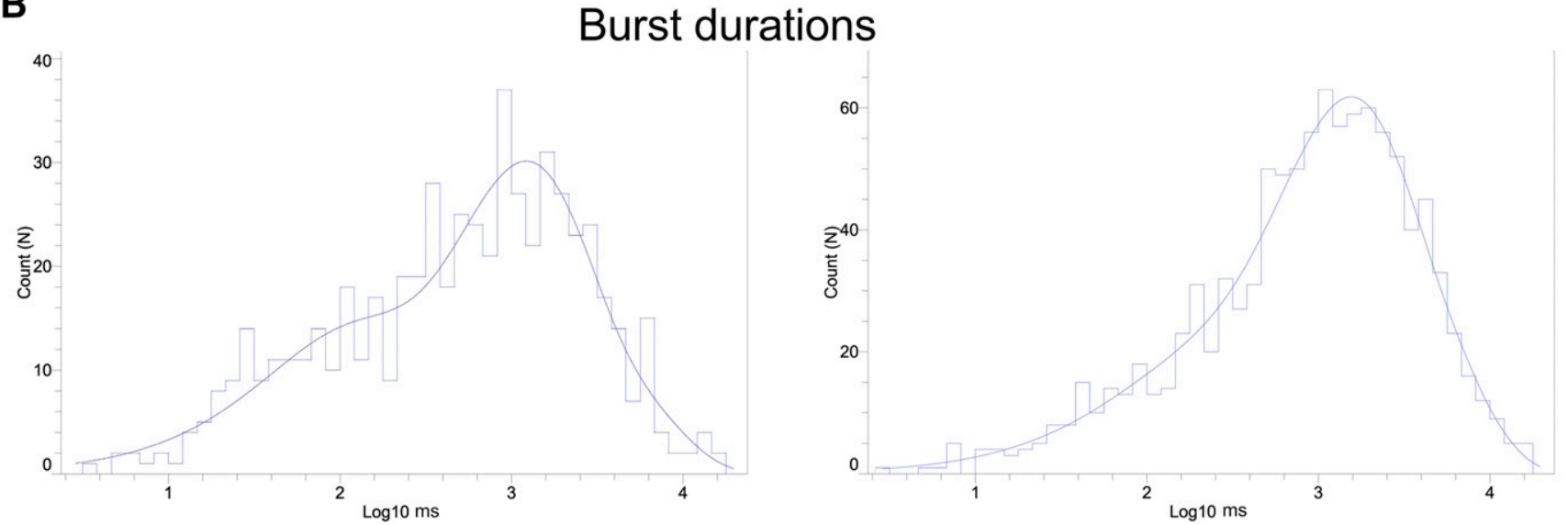

Fig. 6. (A) Representative $\alpha 7$ single-channel traces of cell-attached patch recordings in $10 \mu \mathrm{M}$ GAT107 or $10 \mu \mathrm{M}$ B-973B, shown at different time scales as indicated. Currents were sampled at $50 \mathrm{kHz}$ and low-pass filtered at $6 \mathrm{kHz}$. In the lower right trace, the various conductance levels are indicated: closed channels (C), small subconductance (S), intermediate subconductance (I), and full (large) open (F). (B) Normalized burst duration distributions for 584 bursts in GAT104 and 1047 in B-973B. Bursts were defined, and sequences of single-channel events were separated from other channel activity by a closed time of $>50$ milliseconds. The distributions were best fit by three exponential components. See Table 1 for the fit values.

unchanged, 1849.4 milliseconds. Using this to estimate an offrate of 0.54 second $^{-1}$ for mecamylamine from B-973B-activated channels and the difference in open times to estimate an on-rate of $2,300,000 \mathrm{M}^{-1} \mathrm{~s}^{-1}$, we can further estimate the $K_{\mathrm{d}}$ of mecamylamine as $230 \mathrm{nM}$ for B-973B-activated channels.

In contrast, the effects of mecamylamine in the presence of GAT107 seem more consistent with a parallel block of both open and closed channels (Papke and Oswald, 1989). Averaging across all bursts, in the absence of mecamylamine, open time in GAT107 bursts was 1340.7 milliseconds, and in the presence of mecamylamine, average open time per GAT107 burst was reduced to 277.3 milliseconds.

In Silico Analysis of Ago-PAM Binding and Activity. As discussed previously, the allosteric activation of $\alpha 7$ receptors by ago-PAMs has been proposed to arise from binding

\section{TABLE 1}

Burst duration distributions fit values (see Fig. 6)

$\mathrm{A}_{1}, \mathrm{~A}_{2}$, and $\mathrm{A}_{3}$ refer to the percentage of the events fit to components 1,2 , and 3 , respectively.

\begin{tabular}{ccccccc}
\hline Drug & $\mathrm{A}_{1} \%$ & {$[\tau 1] \mathrm{ms}$} & $\mathrm{A}_{2} \%$ & {$[\tau 2] \mathrm{ms}$} & $\mathrm{A}_{3} \%$ & {$[\tau 3] \mathrm{ms}$} \\
\hline GAT107 & 19 & 74 & 60 & 1058 & 21 & 3560 \\
B-973B & 8 & 86 & 57 & 1224 & 35 & 3330 \\
\hline
\end{tabular}

these agents to both of two different classes of sites, a PAM site in the TMD and a DAA site in the ECD that is, at least in part, distinct from the binding site for $\mathrm{ACh}$ or other orthosteric agonists (Horenstein et al., 2016). Given the large differences in the structure of GAT107 and B-973B (Fig. 3), it is reasonable that they might show very different activities at one or both classes of sites. Although there are no perfect models of $\alpha 7$ structure, significant advancements have been made in recent years toward developing homology models thanks to the resolution of protein structures with higher homology to the $\alpha 7$ receptor (see Materials and Methods) that give some possible insights into the basis for the difference in GAT107 and B-973B activity we observed.

To explore ligand binding poses at the orthosteric and the DAA sites of the ECD of the receptor, we ran 20 nanoseconds molecular dynamics (MD) simulations with a wild-type $\alpha 7$ ECD homology model to refine the amino acid sidechain geometries. The last snapshot of this MD trajectory was minimized and used as the docking model.

We have previously identified a putative DAA binding site in a deep pocket in the vestibule of the receptor (Horenstein et al., 2016). GAT107 and B-973B showed poses at this DAA site, as well as some poses that overlapped the orthosteric binding site. The minimum pharmacophore of an $\alpha 7$ agonist is 


\section{GAT107}
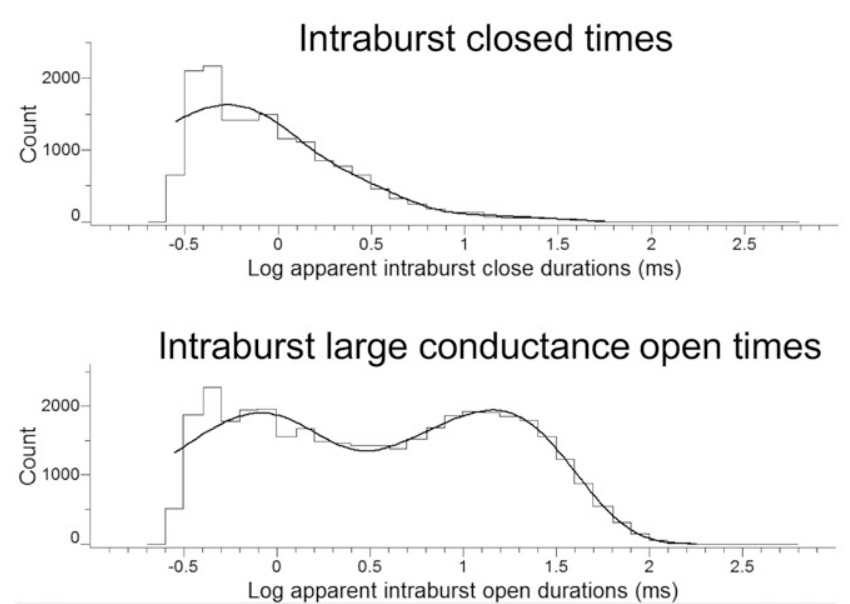

Intraburst intermediate conductance open times
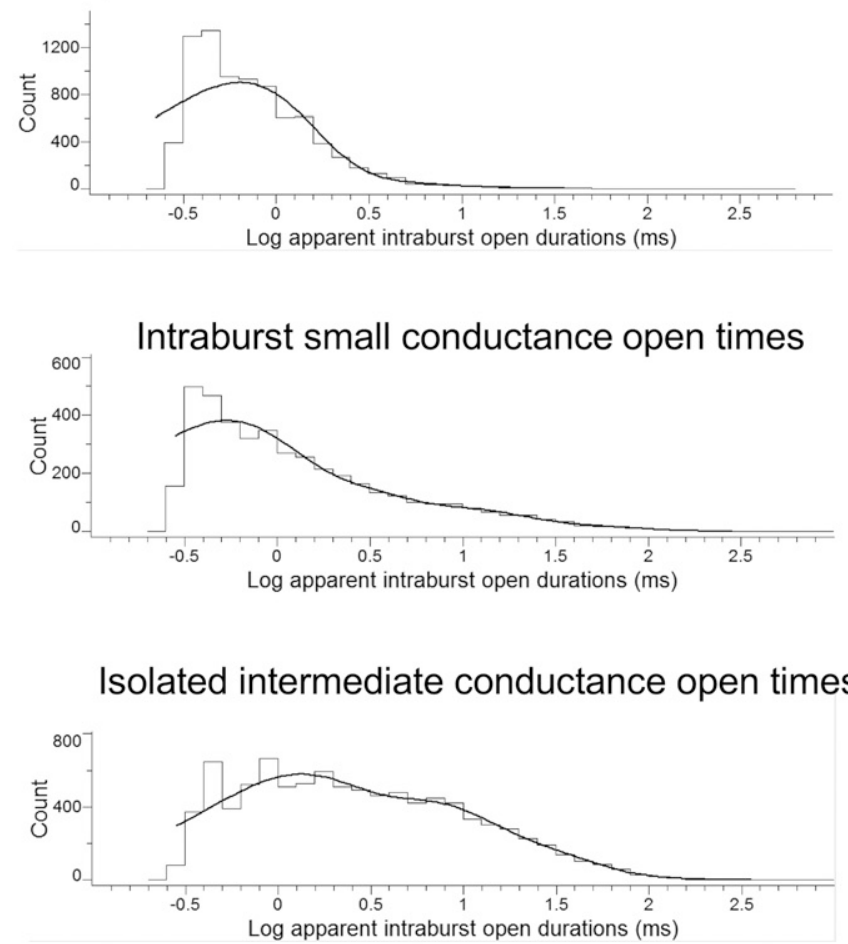

B-973B
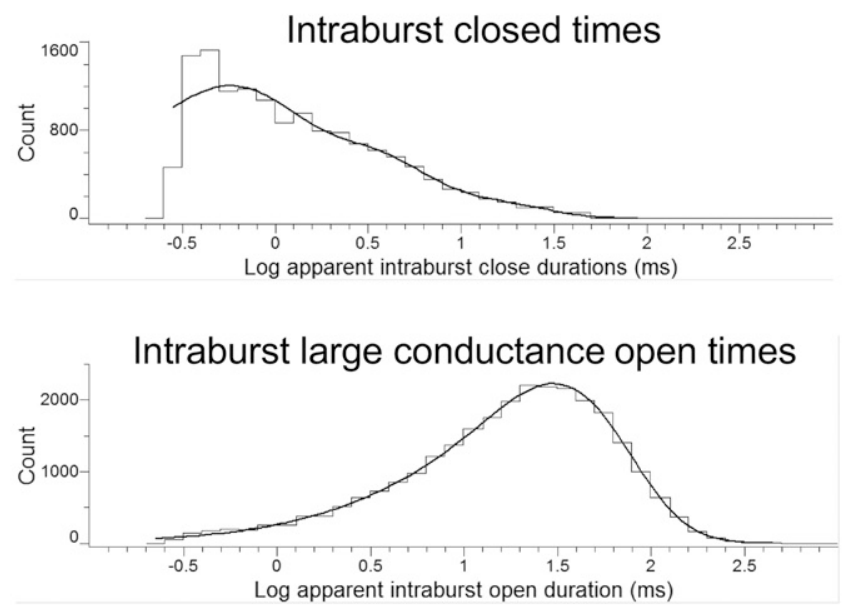

Intraburst intermediate conductance open times

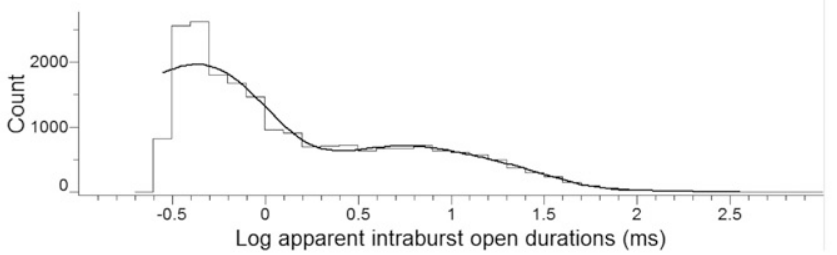

Intraburst small conductance open times

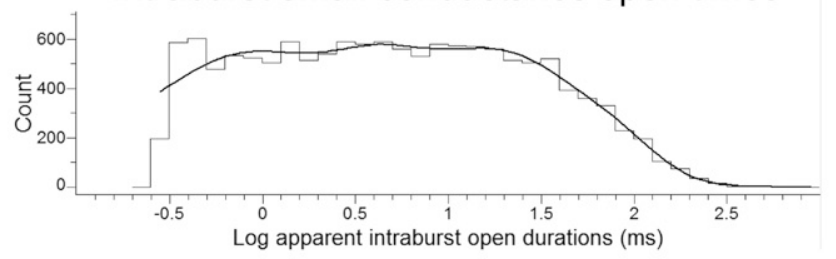

Isolated intermediate conductance open times

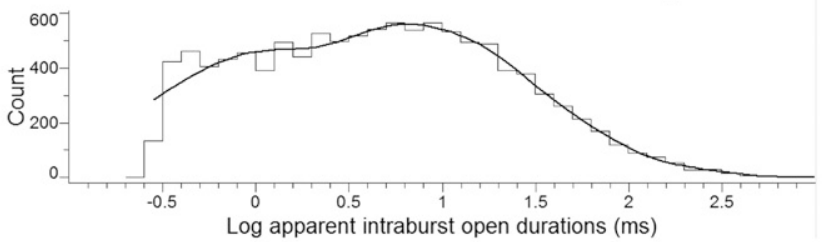

Fig. 7. Intraburst components of single-channel $\alpha 7$ bursts evoked by $10 \mu \mathrm{M}$ GAT107 or $10 \mu \mathrm{M}$ B-973. See Table 2 for the fit values.

a charged nitrogen (Horenstein et al., 2008), which forms $\pi$-cation interactions with the aromatic residues of the orthosteric site (Zhong et al., 1998) and an electrostatic interaction with the backbone oxygen of $\alpha 7 \mathrm{~W} 149$. Other agonists, such as ACh and epibatidine, have their hydrophobic groups buried inside a hydrophobic pocket among residues W149, L109, and L119 and may form additional hydrogen bonds through their heteroatoms; however, because neither GAT107 nor B-973B has a permanently charged nitrogen group, they cannot form the landmark electrostatic interactions with the aromatic residues within the orthosteric site. As a result, the orthosteric poses of these compounds are different from those expected for orthosteric full and partial agonists and so would not be consistent with conventional mechanism for channel activation.

Interestingly, we found that the docking of the ago-PAMs was different at each of the subunit interfaces in our model (Supplemental Fig. 1; Supplemental Table 1). Similar nonequivalence of ACh binding sites has been reported previously in other in silico studies (Henchman et al., 2003; Law et al., 2005 ) and is consistent with studies of large partial agonists crystallized in the AChBP (Hibbs et al., 2009) that showed ligands bound in different orientations at various interfaces. Preferred docking poses for GAT107 and B-973B at one sample interface $\left(I_{\mathrm{BC}}\right)$ are shown in Fig. 10A. At this interface, the GAT107 poses were closest to the orthosteric site, whereas 
TABLE 2

Intraburst statistics

\begin{tabular}{|c|c|c|c|c|}
\hline \multirow{2}{*}{ Type of Intraburst Event } & \multicolumn{2}{|c|}{ GAT107 } & \multicolumn{2}{|c|}{ B-973B } \\
\hline & $\mathrm{A} \%$ & {$[\tau] \mathrm{ms}$} & $\mathrm{A} \%$ & {$[\tau] \mathrm{ms}$} \\
\hline \multirow[t]{3}{*}{ Closures } & $64 \pm 21$ & $0.44 \pm 0.33$ & $55 \pm 9$ & $0.45 \pm 0.22$ \\
\hline & $32 \pm 20$ & $1.61 \pm 0.72$ & $35 \pm 9$ & $2.22 \pm 0.47$ \\
\hline & $4 \pm 0.9$ & $10.5 \pm 2.33$ & $11 \pm 10$ & $10.46 \pm 0.91$ \\
\hline \multirow[t]{5}{*}{ Isolated subconductancs } & $3 \pm 0.5$ & $41.3 \pm 6.77$ & $23 \pm 5$ & $0.58 \pm 0.21$ \\
\hline & $44 \pm 8$ & $0.92 \pm 0.19$ & $21 \pm 14$ & $2.86 \pm 0.65$ \\
\hline & $35 \pm 24$ & $4.83 \pm 0.68$ & $31 \pm 13$ & $9.26 \pm 0.72$ \\
\hline & $18 \pm 34$ & $16.6 \pm 2.51$ & $5 \pm 8$ & $109 \pm 1.23$ \\
\hline & & & $20 \pm 15$ & $28.8 \pm 0.88$ \\
\hline \multirow[t]{4}{*}{ Large openings } & $39 \pm 11$ & $0.64 \pm 0.23$ & $4 \pm 1$ & $2.62 \pm 0.23$ \\
\hline & $8 \pm 10$ & $2.49 \pm 2.18$ & $80 \pm 19$ & $28.2 \pm 0.08$ \\
\hline & $23 \pm 2.2$ & $11.7 \pm 3.20$ & $16 \pm 20$ & $51.3 \pm 0.38$ \\
\hline & $30 \pm 27$ & $18.9 \pm 1.67$ & & \\
\hline \multirow[t]{5}{*}{ Intermediate openings } & $59 \pm 18$ & $0.44 \pm 0.29$ & $23 \pm 04$ & $0.49 \pm 0.22$ \\
\hline & $24 \pm 15$ & $1.58 \pm 0.97$ & $25 \pm 5$ & $2.40 \pm 0.33$ \\
\hline & $13 \pm 11$ & $7.95 \pm 1.45$ & $25 \pm 11$ & $11.6 \pm 0.53$ \\
\hline & $4 \pm 13$ & $32.9 \pm 2.67$ & $26 \pm 14$ & $41.7 \pm 0.83$ \\
\hline & & & $1 \pm 22$ & $110 \pm 9.68$ \\
\hline \multirow[t]{4}{*}{ Small openings } & $1 \pm 0.5$ & $8.47 \pm 30.4$ & $67 \pm 5$ & $0.40 \pm 0.13$ \\
\hline & $92 \pm 39$ & $0.62 \pm 0.36$ & $1 \pm 0.1$ & $52.9 \pm 10.2$ \\
\hline & $6 \pm 4$ & $2.50 \pm 10.5$ & $15 \pm 20$ & $3.54 \pm 1.22$ \\
\hline & & & $17 \pm 16$ & $12.5 \pm 1.51$ \\
\hline
\end{tabular}

B-973B showed a pose in contact with both the orthosteric and putative DAA sites.

The tendency of $\alpha 7$ receptors to convert to nonconducting (desensitized) states at high levels of agonist occupancy (Papke et al., 2000; Uteshev et al., 2002; Williams et al., 2011a) may have to do with a bias toward the induction of specific conformational states associated with binding to a particular nonequivalent interface. Although this is an interesting hypothesis, it is beyond the scope of either existing experimental data or in silico modeling to test. Since we cannot directly interpret the significance of the difference in binding among the subunit interfaces, these observations are described in the Supplemental Data.

To delineate the poses important to the ago-PAM activity, we docked TQS, a type II PAM that does not exhibit DAA, into the same binding sites as the ago-PAMs (Fig. 10B). Poses shared by TQS and GAT107 or B-973B would seem unlikely to be responsible for ago-PAM activity. The TQS poses at $I_{\mathrm{AB}}$, $I_{\mathrm{BC}}$, and $I_{\mathrm{DE}}$ were similar to those of GAT107. At $I_{\mathrm{CD}}$, although TQS and GAT107 both occupied the DAA site, the binding configurations were different. The sulfonamide group of GAT107 was buried inside the hydrophobic pocket of the DAA site, whereas for TQS, this was the naphthalene group. Furthermore, the GAT107 sulfonamide nitrogen formed a hydrogen bond with the backbone of the L92 residue at the receptor subunit-subunit interface, and a similar interaction was observed for B-973B (data not shown), suggesting a possible significance of this interaction for allosteric activation. This residue is, of course, adjacent to Y93, an essential residue for ordinary orthosteric activation, but not allosteric, as indicated by the ago-PAM activity on the Y93C mutant (Fig. 4).

We also used a homology model of the $\alpha 7$ TMD for our docking studies to analyze the differences at the putative PAM binding site (see Materials and Methods). Other studies
(Gill et al., 2011; Newcombe et al., 2018), using Torpedo nAChR structures (Unwin, 2005; Unwin and Fujiyoshi, 2012) as the starting point, have suggested binding sites in the lower regions of the TMD within or between TM helices. For our work, we used the TMDs of the recently resolved $\alpha 4 \beta 2$ structure (Hibbs et al., 2009) as a starting point. We applied an induced-fit approach to allow for interactions between the ligand molecules and TMD residues and generated a maximum of 10 structures for each ligand. Our data suggest that a binding site that puts the ligands in direct contact with the region below the M2-M3 linker is more likely, which seems reasonable, considering the importance of the M2-M3 linker and Cys-loop interactions for gating (Purohit and Auerbach, 2007; Lee et al., 2009). The TMD helices of the subunit rotated and tilted during the MD simulations, consistent with the proposed channel opening motion (Law et al., 2005). Both agoPAMs docked to two different regions close to each other. One was a region situated below the M2-M3 linker of the TMD defined by the residues F253, M254, V257, P269, I271, F275, L215, and C219 of the positive-face subunit and the residues L209, Y210, L213, and N214 of the negative face subunit (Fig. 11A), and the other was between the neighboring M2 helices of the receptor, where ligand binding could block further rotation of the subunit A (Fig. 11B). The best GAT107 poses were under the M2-M3 linker, but some lower-ranking poses appeared between the M2 helices as well. The B-973B poses were more dispersed overall, but two of the best three poses were between the M2 helices, whereas only one was under the M2-M3 linker. Although most of the GAT107 molecule was buried inside the TMD helices, the larger size of B-973B caused a portion of the molecule to stick out and interact with other TMD residues of the neighboring subunits and helices such as Y211, N214, L215, P218, and A258 (Fig. 11B). These additional contacts of B-973B may be the reason behind the differences in the ago-PAM profiles of 
A

$$
\begin{gathered}
10 \mu \mathrm{M} \text { GAT107 } \\
+30 \mu \mathrm{M} \text { Mecamylamine }
\end{gathered}
$$
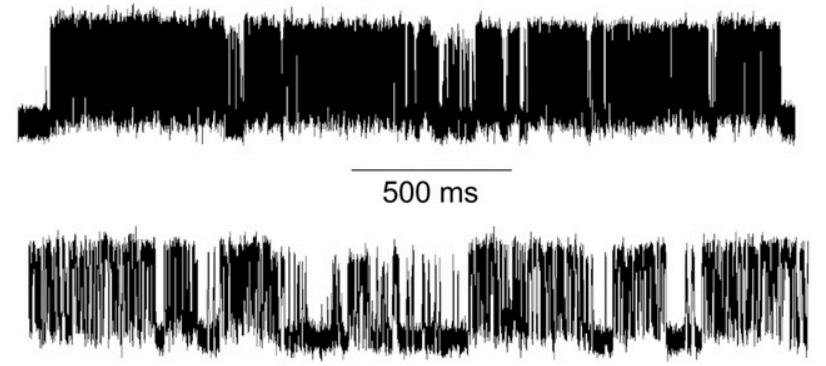

$100 \mathrm{~ms}$

\section{B}

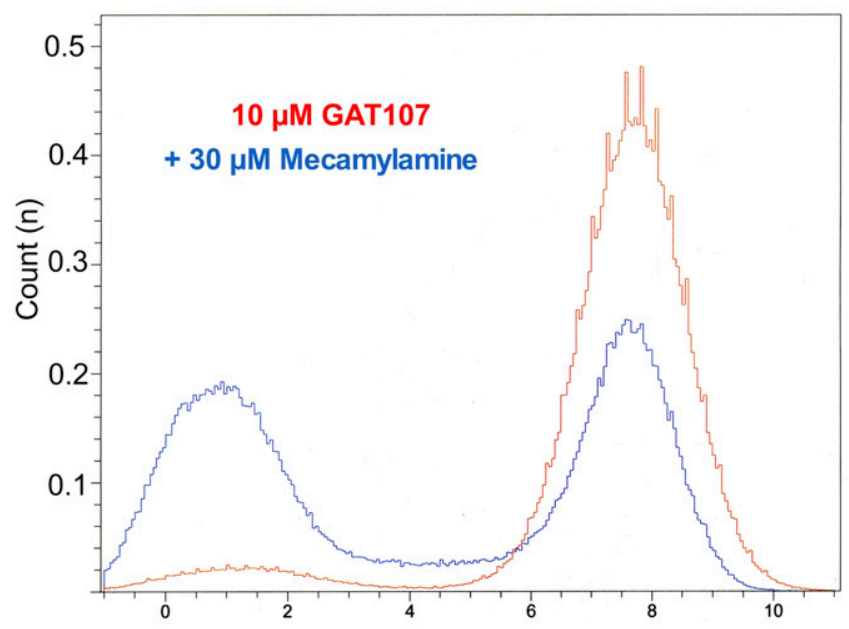

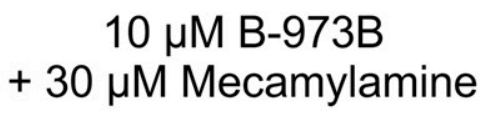
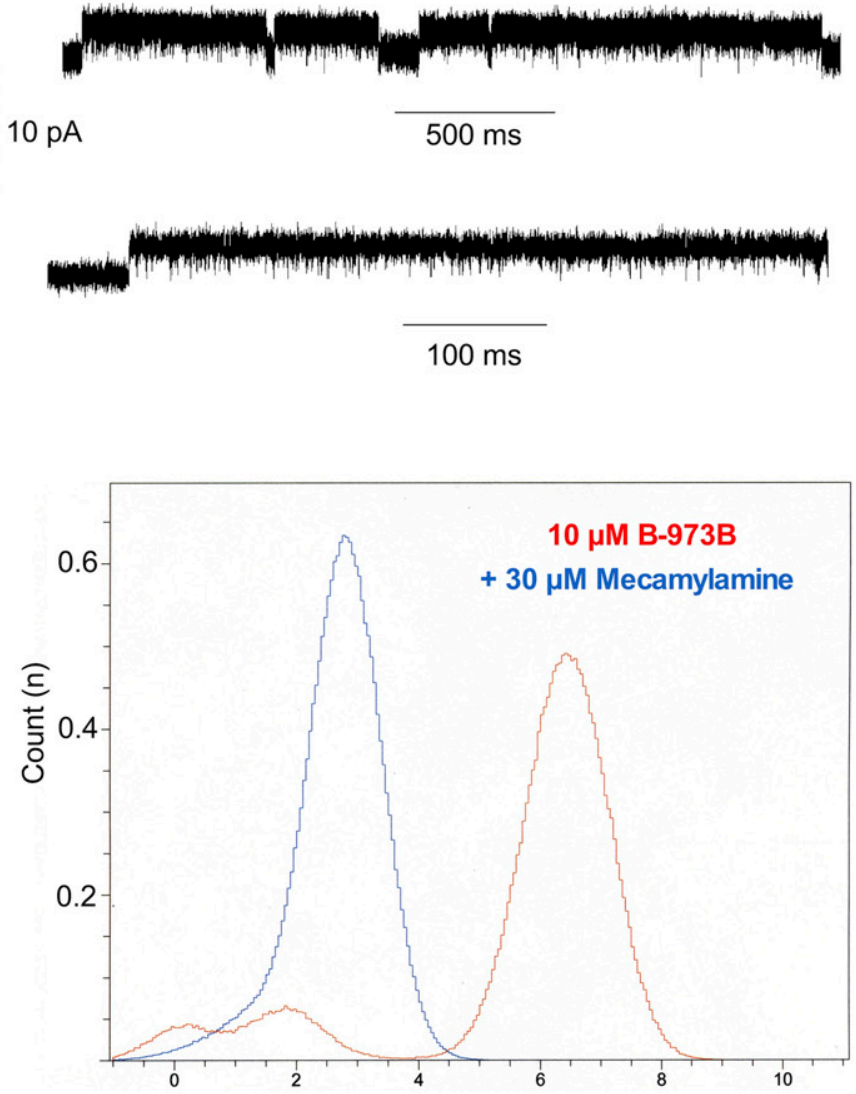

C

Average Log Burst durations

$\star \star \star$

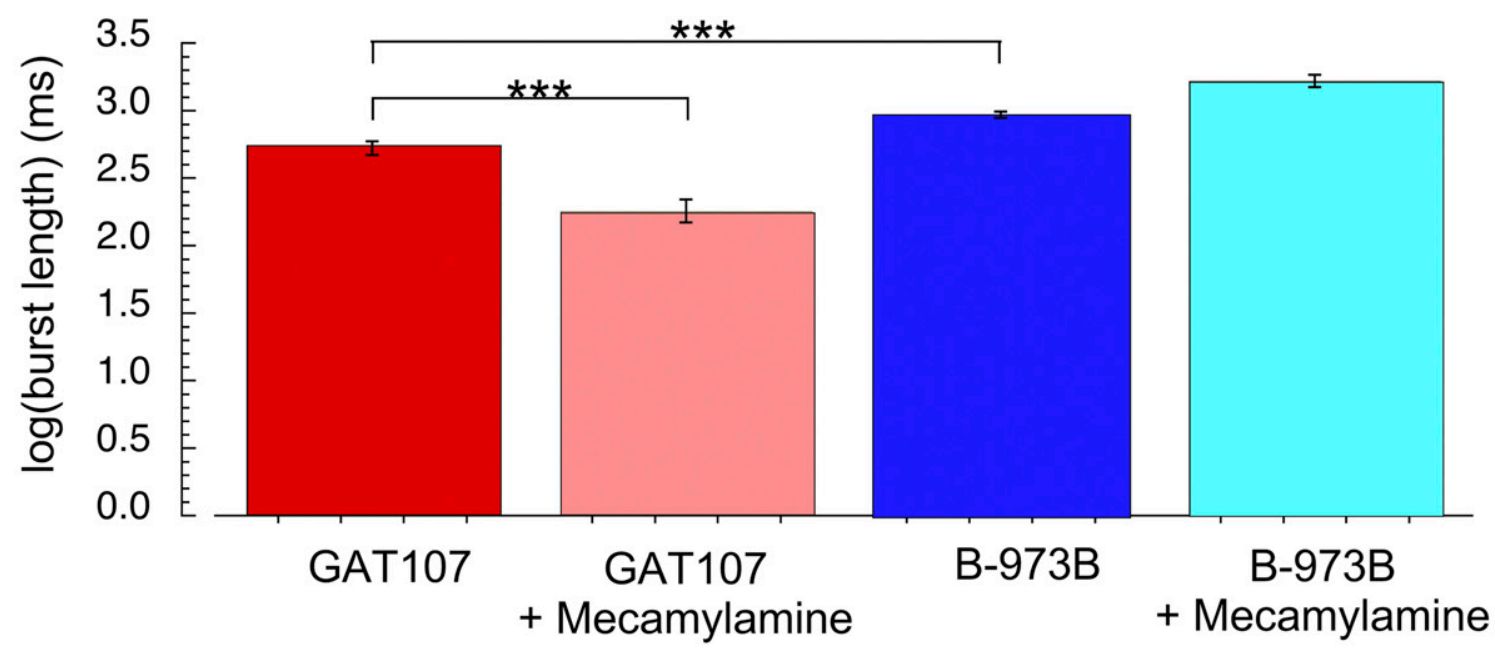

Fig. 8. (A) Representative $\alpha 7$ single-channel traces of cell-attached patches for GAT107 and B-973B coapplied with $30 \mu \mathrm{M}$ mecamylamine shown at different time scales as indicated. Currents were sampled at $50 \mathrm{kHz}$ and low-pass filtered at $6 \mathrm{kHz}$. (B) Representative normalized all points (amplitude) histograms for 1-second intervals of channel-bursting activity in the presence of the ago-PAMs alone (red) or the ago-PAMs plus $30 \mu \mathrm{M}$ mecamylamine (blue). (C) Since burst-duration distributions are exponential functions and unsuitable for parametric comparisons, burst durations were converted to their corresponding $\log _{10}$ values, generating Gaussian-like distributions. Shown are the average $\log _{10}$ burst durations for the two ago-PAMs \pm S.E.M. of those values; $t$ test comparisons indicated significant differences between the values for GAT107 alone compared with B-973B alone, that GAT107 bursts were shorter in mecamylamine, and that B-973B bursts were longer in the presence of mecamylamine. A Bonferroni correction was applied so that $P$ values for a given level of significance are reduce by a factor of 3 . The $P$ value 


\section{$10 \mu \mathrm{M}$ GAT107 + 30 нM Mecamylamine}

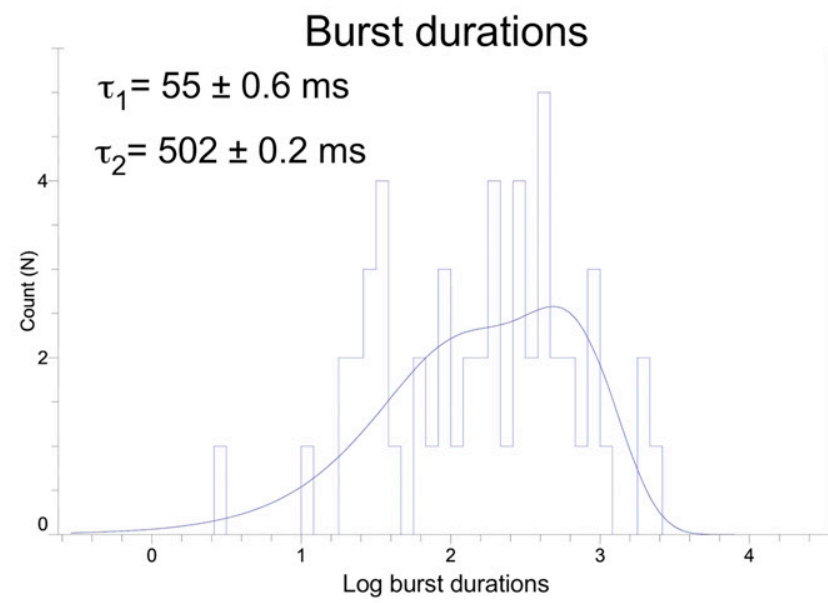

Intraburst closed times

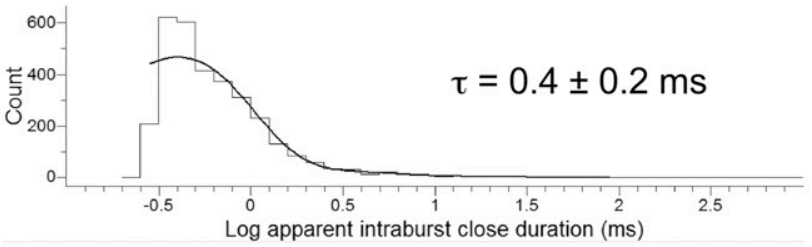

Large conductance open times

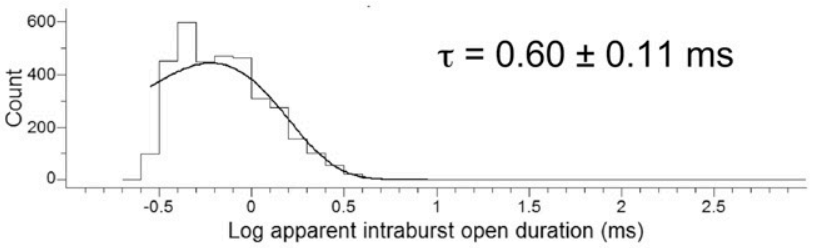

Small conductance open times

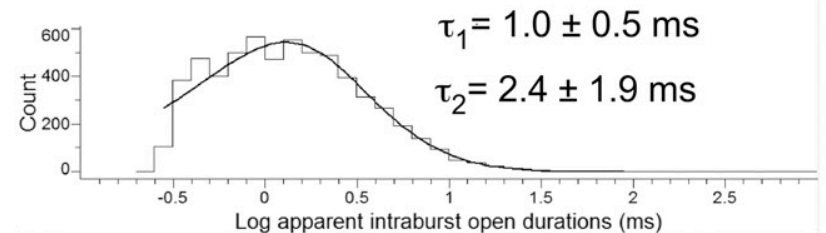

\section{$10 \mu \mathrm{M}$ B-973B \\ $+30 \mu \mathrm{M}$ Mecamylamine}

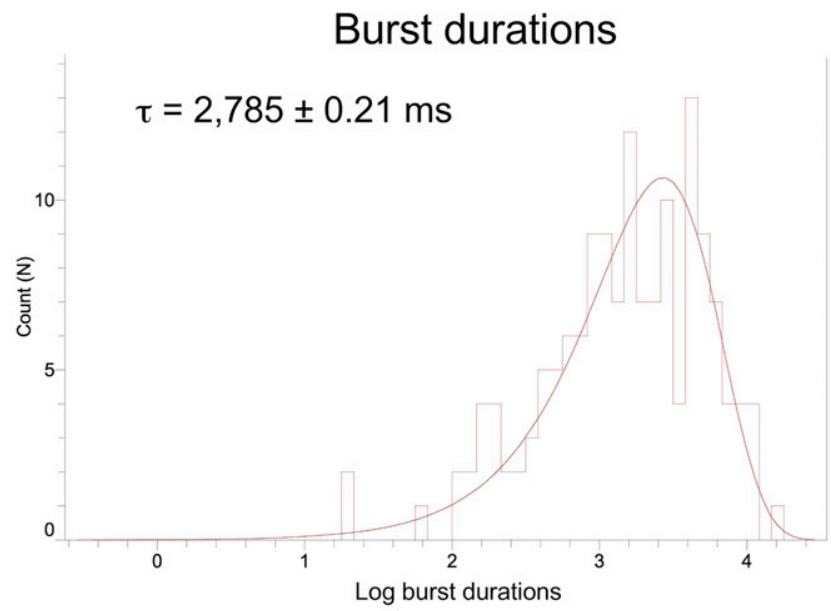

Intraburst closed times

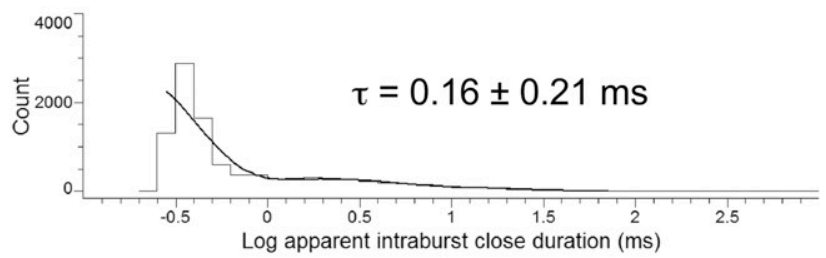

og apparent intraburst close duration (ms)

\section{Small conductance open times}

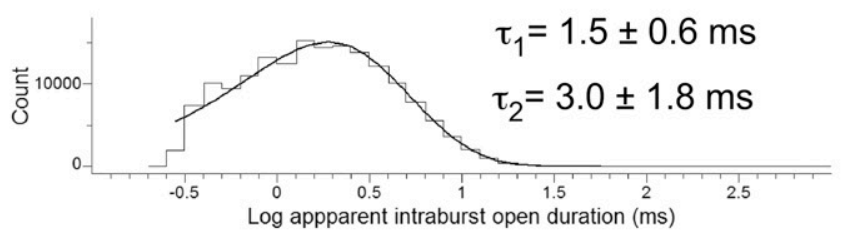

Fig. 9. Kinetic analysis of single-channel $\alpha 7$ activity in bursts evoked by $10 \mu \mathrm{M}$ GAT107 or $10 \mu \mathrm{M}$ B-973B. Fit $\log _{10}$ probability histograms displaying burst durations and apparent open and close durations. Note that in the presence of B-973B and mecamylamine, only a single type of opening was resolved.

B-973B and GAT107, particularly regarding the availability of binding sites within the channel for mecamylamine and possibly other noncompetitive antagonists.
The M254L mutation can affect a variety of type I and type II PAM molecules to different extents (Bertrand et al., 2008; Young et al., 2008; Gill et al., 2011). We made the in silico

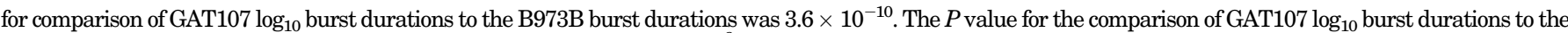

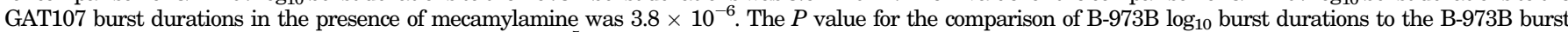

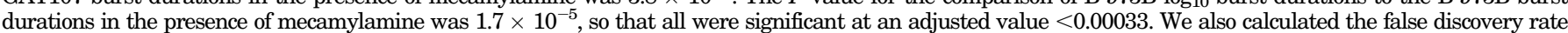

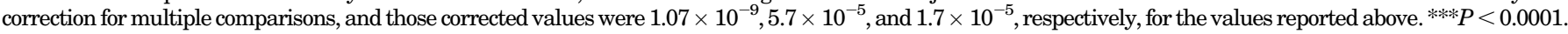


TABLE 3

Burst $\mathrm{P}_{\text {open }}$ comparisons

\begin{tabular}{lcccr}
\hline Condition & Average Open, ms & Average Closed, ms & Popen & Open Time (ms) per Burst \\
\hline $10 \mu$ M GAT107 & 8.13 & 1.79 & 0.82 & 1340.7 \\
$10 \mu$ M B973B & 18.87 & 2.88 & 0.87 & 1854.3 \\
GAT107 + Mec. & 1.70 & 0.89 & 0.66 & 277.3 \\
B973B + Mec. & 2.53 & 1.68 & 0.60 & 1849.4 \\
\hline
\end{tabular}

$\alpha 7 \mathrm{M} 254 \mathrm{~L}$ mutation using UCSF chimera (Pettersen et al., 2004) to see how it affected the interactions among the TMD residues (Fig. 11C). All possible rotamers of L254 had clashes with the nearby F275, which not only restricted the PAM binding pocket but could also cause global disruption of the TMD geometry owing to the compact network of hydrophobic residues at the TMD. GAT107 directly enters the binding pocket without passing through other TMD helices. When B-973B binds to this site, however, it does so like a hook where the difluorinated phenyl ring sits in the hydrophobic pocket, whereas the other part of the molecule passes over M254 and interacts with other TMD residues. Because GAT107 is smaller than B-973B and has fewer interactions in the direction of M254, it is less likely to suffer from a clash with L254, which would explain why the M254L mutation affected B-973B more significantly than GAT107 (Fig. 11D).

\section{Discussion}

Although structurally very different, both GAT107 and B-973B are ago-PAMs, capable of producing allosteric activation when the orthosteric site is disabled by mutations. Both will synergize with orthosteric agonists and are more potent as PAMs than as allosteric agonists, evoking greater channel activation when coapplied with ACh than when applied alone. Both the potentiating effects and allosteric agonism rely on the TMD PAM binding site. Both apparently destabilize desensitized states induced by agonist binding; however, GAT107-potentiating effects appear to be limited by the induction of $\mathrm{D}_{\mathrm{i}}$ (PAM-insensitive desensitization) at high concentrations, accounting for an inverted-U concentrationresponse relationship (Papke et al., 2014b), whereas B-973Bpotentiating effects are less limited at high concentrations, and persistent activation can be evoked, even with limited exposure to ACh (Figs. 2 and 3).

On the microscopic level, both ago-PAMs caused individual $\alpha 7$ receptors to show huge increases in single-channel $P_{\text {open }}$ by manifesting long-lasting bursts, as previously reported for currents promoted by PNU-120596 (Williams et al., 2011b) and other type II PAMs (Andersen et al., 2016). Although ideally it would be best to compare macroscopic and microscopic response in the same system, comparisons of the potentiation of single-channel currents in HEK cells to whole-cell responses in oocytes suggests that, at the concentration studied, more channels were quiescent than activated at any time, permitting the resolution of protracted singlechannel bursts with little or no overlapping activation of other channels. Whereas on the macroscopic level the allosteric activation produced by $10 \mu \mathrm{M}$ B973B had a net charge only twice that stimulated by $60 \mu \mathrm{M}$ ACh (Fig. 2), the total open time within B-973B single-channel bursts (Table 3) was 30,000 -fold greater that of the average $60 \mu \mathrm{M}$ ACh-activated currents (Williams et al., 2011b).
Under our experimental conditions, when only the ago-PAM is in the pipette, we can be confident that we are observing only DAA. Likewise, when we contrast that activity to what is observed when mecamylamine is added, we know that we observed the effects of mecamylamine only on allosterically activated channels.

Both ago-PAMs promote a large conductance state that is similar in amplitude, but far more stable, than that promoted by orthosteric agonists (Williams et al., 2011b). The PAMassociated open state(s) are coupled to unstable "flip states" (Lape et al., 2008), promoting bursting. Burst-duration distributions suggest at least three different bursting states. At the concentration tested, bursts of longer duration were promoted more effectively by B-973B than by GAT107. In addition, both drugs also promoted subconductances connected to the same or to different flip states.

One interesting difference was that B-973B-evoked currents, unlike those evoked by GAT107, were resistant to mecamylamine, a neuronal nAChR-selective channel blocker (Webster et al., 1999). In mecamylamine, GAT107 bursts were fewer, shorter, and contained proportionately more smallconductance openings, suggesting that large or full openings are more easily blocked and that channels do not have to reopen to release the blocker. In contrast, B-973B bursts in the presence of mecamylamine were longer and consisted exclusively of intermediate and small conductance openings. Channels appeared to be prevented from entering the large open state, which may have to do with the size and positioning of B-973B in the TMD site. It is known that sensitivity to mecamylamine and other noncompetitive antagonists is regulated by sequence at sites in the pore-forming M2 domain (see Table 4). Specifically, the residues at the $10^{\prime}$ and $6^{\prime}$ positions (Miller, 1989) in the muscle $\beta 1$ subunit protect muscle receptors (Webster et al., 1999). Perhaps the binding of B-973B in the PAM site partially occludes or otherwise distorts the channel, preventing complete block by mecamylamine. Partial penetration of the channel by mecamylamine may then exclusively permit the subconductance state that is then blocked in a sequential open-state-dependent manner, leading to protracted bursts, as described for QX-222 inhibition of muscle-type receptors (Neher and Steinbach, 1978). The same M2 residues regulate sensitivity to other antagonists such as tetracaine (Papke et al., 2001), and we determined that B-973B currents are also relatively insensitive to $100 \mu \mathrm{M}$ tetracaine (Supplemental Fig. 3).

Although PAMs have been characterized that differ greatly in structure (Williams et al., 2011c), this is the first case for such diversity in ago-PAMs, which are likely to bind at both the PAM site in the TMD and the allosteric site(s) in the ECD. We used in silico methods to test the hypothesis that they shared the same ECD site; however, the docking studies suggested multiple binding modes or sites in the ECD, including the putative DAA site and atypical binding to the 

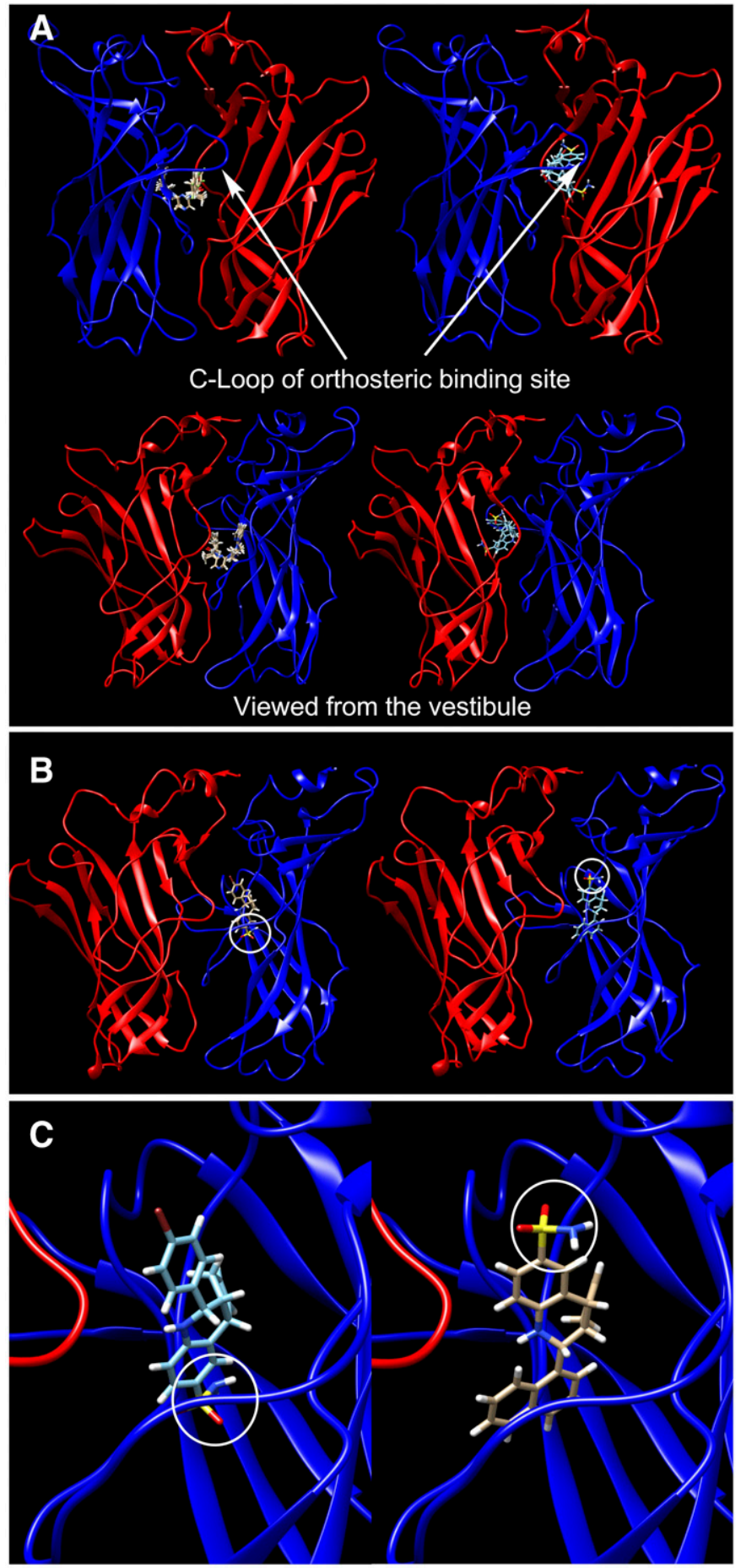

Fig. 10. (A) The top two docking poses of GAT107 (right) and the top four docking poses of B-973 (left) from the outside (top) and the vestibular side (bottom) at $\alpha 7$ ECD I $\mathrm{BC}_{\mathrm{BC}}$. The GAT107 poses shown are at the orthosteric site, whereas the B-973B poses are in contact with both the orthosteric site and the DAA site. (B) The best docking pose of GAT107 (left) and TQS (right) at the $\mathrm{I}_{\mathrm{CD}} \mathrm{DAA}$ site viewed from the vestibular side. The sulfonamide group of each molecule is circled in white. (C) A magnified view of (B).

canonical orthosteric site (Supplemental Data). We know that high levels of binding to the orthosteric sites promote transitions to desensitized states, some of which are perturbed by PAMs, but no data have related this to different binding modes or activity at different interfaces; however, the differences detected in the in silico study may suggest that binding sites at the multiple subunit interfaces may play different roles in coupling ligand binding to specific conformational transitions of the receptor.

Both ago-PAMs produced complex patterns of subconductances and different burst states, unusual for $\mathrm{nAChR}$ in the absence of allosteric modulation but more common for glutamate-gated ion channels (Traynelis et al., 2010). The different open states we observed may be due to different numbers of sites occupied at either of the allosteric sites, or by different poses at nonequivalent sites at the subunit interfaces or elsewhere in the ECD. These factors would be expected to change dynamically throughout the course of a recording since the ligands were used at a subsaturating concentration. It should be noted that the extreme gain-of-function $\alpha 7 \mathrm{L9}^{\prime} \mathrm{T}$ mutation promotes currents very much like a type II PAM and couples the binding of competitive antagonists to channel activation (Bertrand et al., 1997). The presence of this mutation in $\alpha 7$ diminishes the relative effects of PAMs (Williams et al., 2011b; Newcombe et al., 2018), suggesting that the mutation itself partially destabilizes desensitization in a manner similar to a PAM. The distinction between PAMs and ago-PAMs-that PAMs require agonist coapplication whereas ago-PAMs do not, can also be reduced or eliminated by mutations in the W55 residue of $\alpha 7$ (Horenstein et al., 2016; Newcombe et al., 2018), a mutation that also perturbs desensitization produced by conventional agonists (Gay et al., 2008; Williams et al., 2009). It may also be the case that nonconventional binding of the ago-PAMs to the orthosteric site could also couple to activation with the binding of the agents to the TMD PAM site. We observe similar behavior with weak partial agonists in the presence of the PAM PNU120596 (Papke et al., 2014a).

The environment of the channel itself differs in the open states promoted by the two drugs so that current-dependent signaling may also differ, especially regarding the limitations imposed by the $\mathrm{D}_{\mathrm{i}}$ state. We know from earlier work (Peng et al., 2013) that ions and channel blockers affect the PNUpotentiated channel differently from how they affect the "normal" open state. We have shown that mecamylamine affects GAT107- and B-973B-potentiated channels and the B-973B conductance states differently.

Although superficially similar in some respects, the two agents have different balances in their activities at the two allosteric sites and subsequent coupling to the orthosteric site. These differences may have further implications about how the intracellular domains couple to the interactome and subsequent signal transduction. The subtleties of the pharmacologic manipulation of $\alpha 7$ conformational states may have significance beyond the integrated effects of ion channel currents since $\alpha 7 \mathrm{nAChRs}$ have been shown to play a key role in the regulation of inflammatory disease and neuropathic pain through the cholinergic anti-inflammatory pathway (CAP) (Rosas-Ballina and Tracey, 2009). Although this study has focused on the modulation of ion channel activity produced by these ago-PAMs, it remains the case that most channels remain closed for most of the time, and modulation of CAP appears to rely on such nonconducting states (Papke et al., 2015; Horenstein and Papke, 2017). GAT107 has already been shown to be effective in models of neuropathic and inflammatory pain (Bagdas et al., 2016), and preliminary data (Garai et al., 2018) indicate that B-973B has similar effects. 

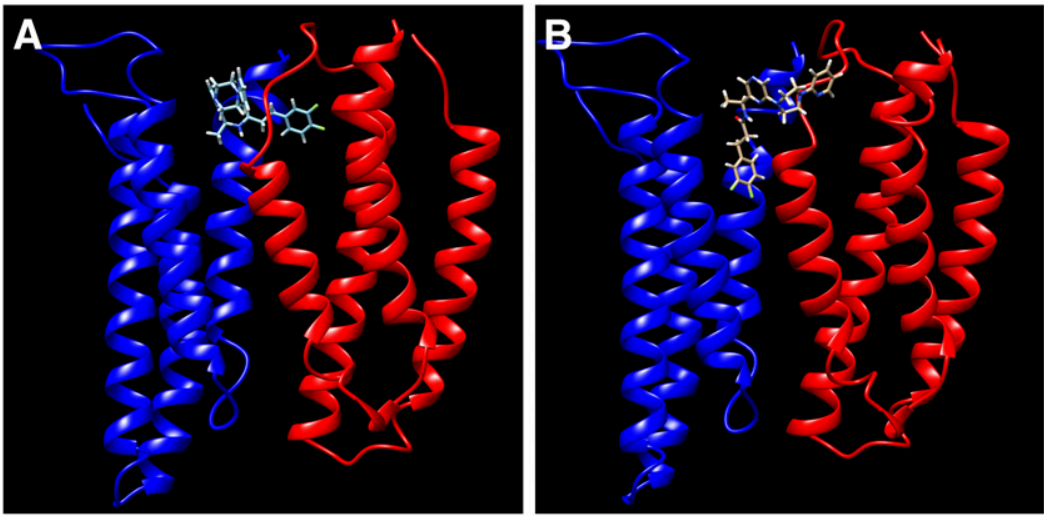

Fig. 11. (A) A TMD binding pose of B-973B, where the difluorinated phenyl ring of the molecule overlaps the putative GAT107 binding site (data not shown) under the M2-M3 linker. (B) A TMD-binding pose of B-973B situated between the M2 helices of two neighboring subunits. (C) A closeup view of the B-973B pose in (A) showing the interactions between B-973B (space-fill) and residues M254 of M2 and F275 of M3 (sticks) (D) The clashes between M2 and M3 residues L254 and F275 and B-973B caused by
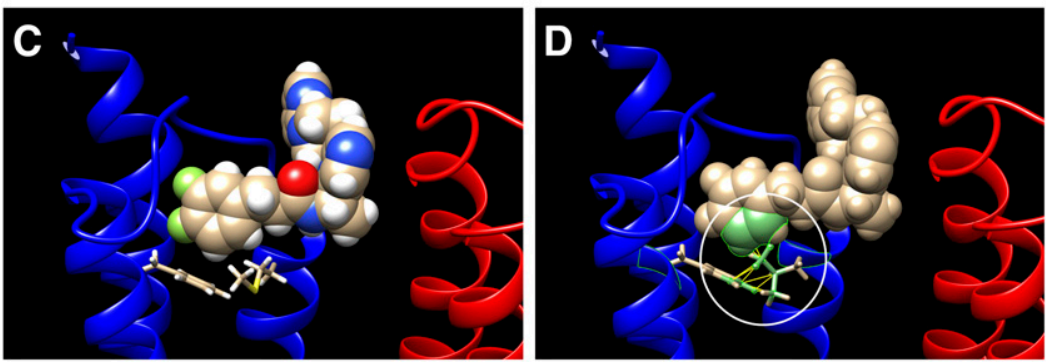
the M254L mutation. Clashing regions of each molecule are circled in white. The clashing atoms of each residue are shown in green, and the individual clashes are shown with yellow lines.

The limitations on $\alpha 7$ channel activation, associated with its homomeric composition, have made it a fascinating and challenging target for drug development (Papke, 2014). Highly selective agonists and partial agonists (Horenstein et al., 2008) were developed in the hopes that they might produce treatments for Alzheimer disease. With the discovery of $\alpha 7$-selective PAMs (Williams et al., 2011c) and more recently, ago-PAMs, the limitations of channel activation have been removed, and the discovery of CAP demonstrated significance for $\alpha 7$ targeting drugs beyond channeldependent processes (de Jonge and Ulloa, 2007). The TQS scaffold on which GAT107 is based has already been shown to be a useful starting point for the development of related agents with diverse properties (Gill-Thind et al., 2015; Horenstein et al., 2016; Newcombe et al., 2018). Characterization of B-973B as a structurally unrelated ago-PAM with unique properties provides an exciting new starting point for further development of potential therapeutic agents.

\section{Acknowledgments}

We thank Alexander den Boef for technical help with the oocyte experiments and Matthew Robinson of The University of Florida Clinical and Translational Science Institute for assistance with

\section{TABLE 4}

Sequence in the second transmembrane domain of select $n A C h R$ subunits with $6^{\prime}$ and $10^{\prime}$ residues in bold

\begin{tabular}{lc}
\hline Domain & Sequence \\
\hline$\alpha 1$ & MTLSISVLLSLTVFLLVIVELIPST \\
$\gamma$ & CTVATNVLLAQTVFLFLVAKKVPET \\
Delta & TSVAISVLLAQSVFLLLISKRLPAT \\
$\alpha 3$ & VTLCISVLLSLTVFLLVITETIPST \\
$\beta 1$ & MGLSIFALLTLTVFLLLLADKVPET \\
$\beta 4$ & MTLCISVLLALTFFLLLISKIVPPT \\
$\beta 2$ & MTLCISVLLALTVFLLLISKIVPPT \\
& $6^{\prime}$ 10' $^{\prime}$ \\
& \\
& ISLGITVLLSLTVFMLLVAEIMPAT \\
& 254 \\
\hline
\end{tabular}

statistics. We also thank Khan Manther for preliminary work with the single channel studies.

\section{Authorship Contributions}

Participated in research design: Quadri, Horenstein, Papke.

Conducted experiments: Quadri, Stokes, Gulsevin.

Contributed new reagents or analytic tools: Garai, Thakur, Gulsevin.

Performed data analysis: Quadri, Gulsevin, Papke.

Wrote or contributed to the writing of the manuscript: Quadri, Thakur, Stokes, Gulsevin, Horenstein, Papke.

\section{References}

Andersen ND, Nielsen BE, Corradi J, Tolosa MF, Feuerbach D, Arias HR, and Bouzat C (2016) Exploring the positive allosteric modulation of human $\alpha 7$ nicotinic receptors from a single-channel perspective. Neuropharmacology 107: 189-200.

Bagdas D, Wilkerson JL, Kulkarni A, Toma W, AlSharari S, Gul Z, Lichtman AH, Papke RL, Thakur GA, and Damaj MI (2016) The $\alpha 7$ nicotinic receptor dual allosteric agonist and positive allosteric modulator GAT107 reverses nociception in mouse models of inflammatory and neuropathic pain. $\mathrm{Br} J$ Pharmacol 173: $2506-2520$

Bertrand D, Bertrand S, Cassar S, Gubbins E, Li J, and Gopalakrishnan M (2008) Positive allosteric modulation of the alpha7 nicotinic acetylcholine receptor: ligand interactions with distinct binding sites and evidence for a prominent role of the M2-M3 segment. Mol Pharmacol 74:1407-1416.

Bertrand S, Devillers-Thiéry A, Palma E, Buisson B, Edelstein SJ, Corringer PJ, Changeux JP, and Bertrand D (1997) Paradoxical allosteric effects of competitive inhibitors on neuronal alpha7 nicotinic receptor mutants. Neuroreport 8: 3591-3596.

Clarke PBS, Schwartz RD, Paul SM, Pert CB, and Pert A (1985) Nicotinic binding in rat brain: autoradiographic comparison of $\left[{ }^{3} \mathrm{H}\right]$ acetylcholine, $\left[{ }_{3} \mathrm{H}\right]$ nicotine, and $\left.{ }^{125} \mathrm{I}\right]$-alpha-bungarotoxin. J Neurosci 5:1307-1315.

Couturier S, Bertrand D, Matter JM, Hernandez MC, Bertrand S, Millar N, Valera S, Barkas T, and Ballivet M (1990) A neuronal nicotinic acetylcholine receptor subunit (alpha 7) is developmentally regulated and forms a homo-oligomeric channel blocked by alpha-BTX. Neuron 5:847-856

de Jonge WJ and Ulloa L (2007) The alpha7 nicotinic acetylcholine receptor as a pharmacological target for inflammation. Br J Pharmacol 151:915-929.

Friesner RA, Murphy RB, Repasky MP, Frye LL, Greenwood JR, Halgren TA, Sanschagrin PC, and Mainz DT (2006) Extra precision glide: docking and scoring incorporating a model of hydrophobic enclosure for protein-ligand complexes. J Med Chem 49:6177-6196.

Frisch MJ, Trucks GW, Schlegel HB, Scuseria GE, Robb MA, Cheeseman JR, Scalmani G, Barone V, Petersson GA, Nakatsuji H, et al. (2016) Gaussian 16, Gaussian, Inc., Wallingford, CT.

Garai S, Raja KS, Papke RL, Deschamps J, Damaj MI, and Thakur GA (2018) B-973 a novel ago-PAM of the $\alpha 7$ nicotinic acetylcholine receptor: total synthesis, enantiomer separation and electrophysiological characterization, asymmetric synthesis and in vivo evaluation of the bioactive enantiomer. Med Chem Lett, in press. 
Gay EA, Giniatullin R, Skorinkin A, and Yakel JL (2008) Aromatic residues at position 55 of rat alpha7 nicotinic acetylcholine receptors are critical for maintaining rapid desensitization. $J$ Physiol 586:1105-1115.

Gill JK, Dhankher P, Sheppard TD, Sher E, and Millar NS (2012) A series of $\alpha 7$ nicotinic acetylcholine receptor allosteric modulators with close chemical similarity but diverse pharmacological properties. Mol Pharmacol 81:710-718.

Gill JK, Savolainen M, Young GT, Zwart R, Sher E, and Millar NS (2011) Agonist activation of alpha7 nicotinic acetylcholine receptors via an allosteric transmembrane site. Proc Natl Acad Sci USA 108:5867-5872.

Gill-Thind JK, Dhankher P, D’Oyley JM, Sheppard TD, and Millar NS (2015) Structurally similar allosteric modulators of $\alpha 7$ nicotinic acetylcholine receptors exhibit five distinct pharmacological effects. J Biol Chem 290:3552-3562.

Grønlien JH, Håkerud M, Ween H, Thorin-Hagene K, Briggs CA, Gopalakrishnan M, and Malysz J (2007) Distinct profiles of alpha7 nAChR positive allosteric modulation revealed by structurally diverse chemotypes. Mol Pharmacol 72:715-724.

Halevi S, Yassin L, Eshel M, Sala F, Sala S, Criado M, and Treinin M (2003) Conservation within the RIC-3 gene family. Effectors of mammalian nicotinic acetylcholine receptor expression. J Biol Chem 278:34411-34417.

Henchman RH, Wang HL, Sine SM, Taylor P, and McCammon JA (2003) Asymmetric structural motions of the homomeric alpha7 nicotinic receptor ligand binding domain revealed by molecular dynamics simulation. Biophys $J \mathbf{8 5}$ 3007-3018.

Hibbs RE, Sulzenbacher G, Shi J, Talley TT, Conrod S, Kem WR, Taylor P, Marchot $\mathrm{P}$, and Bourne Y (2009) Structural determinants for interaction of partial agonists with acetylcholine binding protein and neuronal alpha7 nicotinic acetylcholine receptor. EMBO J 28:3040-3051.

Horenstein NA, Leonik FM, and Papke RL (2008) Multiple pharmacophores for the selective activation of nicotinic alpha7-type acetylcholine receptors. Mol Pharmacol 74:1496-1511.

Horenstein NA and Papke RL (2017) Anti-inflammatory silent agonists. ACS Med Chem Lett 8:989-991.

Horenstein NA, Papke RL, Kulkarni AR, Chaturbhuj GU, Stokes C, Manther K, and Thakur GA (2016) Critical molecular determinants of $\alpha 7$ nicotinic acetylcholine receptor allosteric activation: separation of direct allosteric activation and positive allosteric modulation. J Biol Chem 291:5049-5067.

Jacobson MP, Friesner RA, Xiang Z, and Honig B (2002) On the role of the crystal environment in determining protein side-chain conformations. J Mol Biol $\mathbf{3 2 0}$ $597-608$

Jacobson MP, Pincus DL, Rapp CS, Day TJ, Honig B, Shaw DE, and Friesner RA (2004) A hierarchical approach to all-atom protein loop prediction. Proteins 55: 351-367.

Jakalian A, Jack DB, and Bayly CI (2002) Fast, efficient generation of high-quality atomic charges. AM1-BCC model: II. Parameterization and validation. J Comput Chem 23:1623-1641.

Jones S, Sudweeks S, and Yakel JL (1999) Nicotinic receptors in the brain: correlating physiology with function. Trends Neurosci 22:555-561.

Kulkarni AR and Thakur GA (2013) Microwave-assisted expeditious and efficient synthesis of cyclopentene ring-fused tetrahydroquinoline derivatives using threecomponent povarov reaction. Tetrahedron Lett 54:6592-6595.

Lape R, Colquhoun D, and Sivilotti LG (2008) On the nature of partial agonism in the nicotinic receptor superfamily. Nature 454:722-727.

Law RJ, Henchman RH, and McCammon JA (2005) A gating mechanism proposed from a simulation of a human alpha7 nicotinic acetylcholine receptor. Proc Natl Acad Sci USA 102:6813-6818.

Lee WY, Free CR, and Sine SM (2009) Binding to gating transduction in nicotinic receptors: Cys-loop energetically couples to pre-M1 and M2-M3 regions. J Neurosci 29:3189-3199.

Li SX, Huang S, Bren N, Noridomi K, Dellisanti CD, Sine SM, and Chen L (2011) Ligand-binding domain of an $\alpha 7$-nicotinic receptor chimera and its complex with agonist. Nat Neurosci 14:1253-1259.

Lomize AL, Pogozheva ID, and Mosberg HI (2011) Anisotropic solvent model of the lipid bilayer. 2. Energetics of insertion of small molecules, peptides, and proteins in membranes. J Chem Inf Model 51:930-946.

Maier JA, Martinez C, Kasavajhala K, Wickstrom L, Hauser KE, and Simmerling C (2015) ff14SB: improving the accuracy of protein side chain and backbone parameters from ff99SB. J Chem Theory Comput 11:3696-3713.

Miller C (1989) Genetic manipulation of ion channels: a new approach to structure and mechanism. Neuron 2:1195-1205.

Morales-Perez CL, Noviello CM, and Hibbs RE (2016) X-ray structure of the human $\alpha 4 \beta 2$ nicotinic receptor. Nature 538:411-415.

Neher E and Steinbach JH (1978) Local anaesthetics transiently block currents through single acetylcholine-receptor channels. J Physiol 277:153-176.

Newcombe J, Chatzidaki A, Sheppard TD, Topf M, and Millar NS (2018) Diversity of nicotinic acetylcholine receptor positive allosteric modulators revealed by mutagenesis and a revised structural model. Mol Pharmacol 93:128-140.

Pałczyńska MM, Jindrichova M, Gibb AJ, and Millar NS (2012) Activation of $\alpha 7$ nicotinic receptors by orthosteric and allosteric agonists: influence on singlechannel kinetics and conductance. Mol Pharmacol 82:910-917.

Papke RL (2014) Merging old and new perspectives on nicotinic acetylcholine receptors. Biochem Pharmacol 89:1-11.

Papke RL, Bagdas D, Kulkarni AR, Gould T, AlSharari SD, Thakur GA, and Damaj MI (2015) The analgesic-like properties of the alpha7 nAChR silent agonist NS6740 is associated with non-conducting conformations of the receptor. Neuropharmacology 91:34-42.

Papke RL, Chojnacka K, and Horenstein NA (2014a) The minimal pharmacophore for silent agonism of the $\alpha 7$ nicotinic acetylcholine receptor [published correction appears in J Pharmacol Exp Ther (2015) 354:483]. J Pharmacol Exp Ther 350 $665-680$

Papke RL, Horenstein BA, and Placzek AN (2001) Inhibition of wild-type and mutant neuronal nicotinic acetylcholine receptors by local anesthetics. Mol Pharmacol 60:1365-1374.
Papke RL, Horenstein NA, Kulkarni AR, Stokes C, Corrie LW, Maeng CY, and Thakur GA (2014b) The activity of GAT107, an allosteric activator and positive modulator of $\alpha 7$ nicotinic acetylcholine receptors (nAChR), is regulated by aromatic amino acids that span the subunit interface. J Biol Chem 289:4515-4531.

Papke RL, Meyer E, Nutter T, and Uteshev VV (2000) Alpha7 receptor-selective agonists and modes of alpha7 receptor activation. Eur J Pharmacol $\mathbf{3 9 3}$ 179-195.

Papke RL and Oswald RE (1989) Mechanisms of noncompetitive inhibition of acetylcholine-induced single-channel currents. J Gen Physiol 93:785-811.

Papke RL and Porter Papke JK (2002) Comparative pharmacology of rat and human alpha7 $\mathrm{nAChR}$ conducted with net charge analysis. $\mathrm{Br} J$ Pharmacol 137:49-61.

Papke RL and Stokes C (2010) Working with OpusXpress: methods for high volume oocyte experiments. Methods 51:121-133.

Papke RL, Stokes C, Damaj MI, Thakur GA, Manther K, Treinin M, Bagdas D Kulkarni AR, and Horenstein NA (2018) Persistent activation of $\alpha 7$ nicotinic ACh receptors associated with stable induction of different desensitized states. $\mathrm{Br} J$ Pharmacol 175:1838-1854.

Papke RL, Stokes C, Williams DK, Wang J, and Horenstein NA (2011) Cysteine accessibility analysis of the human alpha7 nicotinic acetylcholine receptor ligandbinding domain identifies L119 as a gatekeeper. Neuropharmacology 60:159-171.

Papke RL and Thinschmidt JS (1998) The correction of alpha7 nicotinic acetylcholine receptor concentration-response relationships in Xenopus oocytes. Neurosci Lett 256:163-166.

Peng C, Kimbrell MR, Tian C, Pack TF, Crooks PA, Fifer EK, and Papke RL (2013) Multiple modes of $\alpha 7 \mathrm{nAChR}$ noncompetitive antagonism of control agonist-evoked and allosterically enhanced currents. Mol Pharmacol 84 $459-475$.

Pettersen EF, Goddard TD, Huang CC, Couch GS, Greenblatt DM, Meng EC, and Ferrin TE (2004) UCSF Chimera-a visualization system for exploratory research and analysis. J Comput Chem 25:1605-1612.

Post-Munson DJ, Pieschl RL, Molski TF, Graef JD, Hendricson AW, Knox RJ, McDonald IM, Olson RE, Macor JE, Weed MR, et al. (2017) B-973, a novel piperazine positive allosteric modulator of the $\alpha 7$ nicotinic acetylcholine receptor. Eur $J$ Pharmacol 799:16-25.

Purohit P and Auerbach A (2007) Acetylcholine receptor gating at extracellular transmembrane domain interface the "pre-M1" linker. J Gen Physiol 130:559-568.

Qin F (2004) Restoration of single-channel currents using the segmental k-mean method based on hidden Markov modeling. Biophys J 86:1488-1501.

Rosas-Ballina M and Tracey KJ (2009) Cholinergic control of inflammation. J Intern Med 265:663-679.

Schaftenaar G and Noordik JH (2000) Molden: a pre- and post-processing program for molecular and electronic structures. J Comput Aided Mol Des 14: $123-134$

Thakur GA, Kulkarni AR, Deschamps JR, and Papke RL (2013) Expeditious synthesis, enantiomeric resolution, and enantiomer functional characterization of (4-(4-bromophenyl)-3a,4,5,9b-tetrahydro-3H-cyclopenta[c]quinoline-8-sulfonamide (4BPTQS): an allosteric agonist-positive allosteric modulator of $\alpha 7$ nicotinic acetylcholine receptors. J Med Chem 56:8943-8947.

Traynelis SF, Wollmuth LP, McBain CJ, Menniti FS, Vance KM, Ogden KK, Hansen KB, Yuan H, Myers SJ, and Dingledine R (2010) Glutamate receptor ion channels: structure, regulation, and function. Pharmacol Rev 62:405-496.

Unwin N (2005) Refined structure of the nicotinic acetylcholine receptor at $4 \mathrm{~A}$ resolution. J Mol Biol 346:967-989.

Unwin N and Fujiyoshi Y (2012) Gating movement of acetylcholine receptor caught by plunge-freezing. $J$ Mol Biol 422:617-634.

Uteshev VV, Meyer EM, and Papke RL (2002) Activation and inhibition of native neuronal alpha-bungarotoxin-sensitive nicotinic ACh receptors. Brain Res 948 $33-46$.

Wada E, Wada K, Boulter J, Deneris E, Heinemann S, Patrick J, and Swanson LW (1989) Distribution of alpha 2, alpha 3, alpha 4, and beta 2 neuronal nicotinic receptor subunit mRNAs in the central nervous system: a hybridization histochemical study in the rat. $J$ Comp Neurol 284:314-335.

Wada K, Ballivet M, Boulter J, Connolly J, Wada E, Deneris ES, Swanson LW, Heinemann S, and Patrick J (1988) Functional expression of a new pharmacological subtype of brain nicotinic acetylcholine receptor. Science 240:330-334.

Wang J, Wolf RM, Caldwell JW, Kollman PA, and Case DA (2004) Development and testing of a general amber force field. J Comput Chem 25:1157-1174.

Webster JC, Francis MM, Porter JK, Robinson G, Stokes C, Horenstein B, and Papke RL (1999) Antagonist activities of mecamylamine and nicotine show reciprocal dependence on beta subunit sequence in the second transmembrane domain. Br J Pharmacol 127:1337-1348.

Williams DK, Peng C, Kimbrell MR, and Papke RL (2012) Intrinsically low open probability of $\alpha 7$ nicotinic acetylcholine receptors can be overcome by positive allosteric modulation and serum factors leading to the generation of excitotoxic currents at physiological temperatures. Mol Pharmacol 82:746-759.

Williams DK, Stokes C, Horenstein NA, and Papke RL (2009) Differential regulation of receptor activation and agonist selectivity by highly conserved tryptophans in the nicotinic acetylcholine receptor binding site. J Pharmacol Exp Ther 330:40-53.

Williams DK, Stokes C, Horenstein NA, and Papke RL (2011a) The effective opening of nicotinic acetylcholine receptors with single agonist binding sites. $J$ Gen Physiol 137:369-384.

Williams DK, Wang J, and Papke RL (2011b) Investigation of the molecular mechanism of the $\alpha 7$ nicotinic acetylcholine receptor positive allosteric modulator PNU120596 provides evidence for two distinct desensitized states. Mol Pharmacol 80 1013-1032.

Williams DK, Wang J, and Papke RL (2011c) Positive allosteric modulators as an approach to nicotinic acetylcholine receptor-targeted therapeutics: advantages and limitations. Biochem Pharmacol 82:915-930. 
Wu EL, Cheng X, Jo S, Rui H, Song KC, Dávila-Contreras EM, Qi Y, Lee J, MonjeGalvan V, Venable RM, et al. (2014) CHARMM-GUI membrane builder toward realistic biological membrane simulations. J Comput Chem 35:1997-2004.

Young GT, Zwart R, Walker AS, Sher E, and Millar NS (2008) Potentiation of alpha7 nicotinic acetylcholine receptors via an allosteric transmembrane site. Proc Natl Acad Sci USA 105:14686-14691.

Zhang ZW, Vijayaraghavan S, and Berg DK (1994) Neuronal acetylcholine receptors that bind alpha-bungarotoxin with high affinity function as ligand-gated ion channels. Neuron 12:167-177.
Zhong W, Gallivan JP, Zhang Y, Li L, Lester HA, and Dougherty DA (1998) From ab initio quantum mechanics to molecular neurobiology: a cation-pi binding site in the nicotinic receptor. Proc Natl Acad Sci USA 95:12088-12093.

Address correspondence to: Roger L. Papke, Department of Pharmacology and Therapeutics, University of Florida, P.O. Box 100267, Gainesville, FL 32610-0267. E-mail: rlpapke@ufl.edu 